\title{
Comprehensive Registry of Esophageal Cancer in Japan, 2009
}

\author{
Yuji Tachimori $^{1} \cdot$ Soji Ozawa $^{2} \cdot$ Hodaka Numasaki $^{3} \cdot$ Mitsuhiro Fujishiro $^{4}$. \\ Hisahiro Matsubara ${ }^{5} \cdot$ Tsuneo Oyama $^{6} \cdot$ Masayuki Shinoda $^{7} \cdot$ Yasushi Toh $^{8}$. \\ Harushi Udagawa ${ }^{9} \cdot$ Takashi Uno $^{10}$ - The Registration Committee for Esophageal \\ Cancer of the Japan Esophageal Society
}

Received: 7 February 2016 / Accepted: 14 March 2016 / Published online: 29 March 2016

(c) The Author(s) 2016. This article is published with open access at Springerlink.com

\section{Preface 2009}

We deeply appreciate the great contributions of many physicians in the registry of esophageal cancer cases. The Comprehensive Registry of Esophageal Cancer in Japan, 2009 was published here, despite some delay. The registry complies with the Act for the Protection of Personal Information. The encryption with a HASH function is used for "anonymity in an unlinkable fashion".

We briefly summarized the Comprehensive Registry of Esophageal Cancer in Japan, 2009. Japanese Classification of Esophageal Cancer 10th and UICC TNM Classification 6th were used for cancer staging according to the subjected year. A total of 6260 cases were registered from

These data were first made available on January 2016, as the Comprehensive Registry of Esophageal Cancer in Japan, 2009. Not all the tables and figures are reprinted here.

The authors were members of the Registration Committee for Esophageal Cancer, the Japan Esophageal Society, and made great contributions to the preparation of this material.

\section{Yuji Tachimori}

ytachimo@ncc.go.jp

1 Esophageal Surgery Division, National Cancer Center Hospital, 5-1-1 Tsukiji, Chuo-ku, Tokyo 104-0045, Japan

2 Department of Gastroenterological Surgery, Tokai University School of Medicine, Isehara, Japan

3 Department of Medical Physics and Engineering, Osaka University Graduate School of Medicine, Osaka, Japan

4 Department of Endoscopy and Endoscopic Surgery, Graduate School of Medicine, University of Tokyo, Tokyo, Japan

5 Department of Frontier Surgery, Graduate School of Medicine, Chiba University, Chiba, Japan
276 institutions in Japan. Tumor locations were cervical: $4.4 \%$, upper thoracic: $11.9 \%$, middle thoracic: $48.0 \%$, lower thoracic: $27.7 \%$ and EG junction: $6.6 \%$. Superficial carcinomas (Tis, T1a, T1b) were $36.7 \%$. As for the histologic type of biopsy specimens, squamous cell carcinoma and adenocarcinoma accounted for 90.5 and $3.8 \%$, respectively. Regarding clinical results, the 5-year survival rates of patients treated using endoscopic mucosal resection, concurrent chemoradiotherapy, radiotherapy alone, chemotherapy alone, or esophagectomy were 86.2, 27.9, 20.2, 5.8, and $55.9 \%$, respectively. Esophagectomy was performed in 3844 cases. Concerning the approach used for esophagectomy, $24.9 \%$ of the cases were treated thoracoscopically. The operative mortality (within 30 days after surgery) was $1.01 \%$ and the hospital mortality was $4.76 \%$.

We hope that this Comprehensive Registry of Esophageal Cancer in Japan, 2009 will help to improve all aspects of the diagnosis and treatment of esophageal cancer in Japan.

6 Department of Gastroenterology, Saku General Hospital, Nagano, Japan

7 Department of Thoracic Surgery, Aichi Cancer Center Hospital, Aichi, Japan

8 Department of Gastroenterological Surgery, National Kyushu Cancer Center, Fukuoka, Japan

9 Department of Gastroenterological Surgery, Toranomon Hospital, Tokyo, Japan

10 Department of Radiology, Graduate School of Medicine, Chiba University, Chiba, Japan 


\section{Contents}

I. Clinical factors of esophageal cancer patients treated in 2009

1. Institution-registered cases in $\mathbf{2 0 0 9}$

2. Patient Background

Table 1 Age and gender

Table 2 Primary treatment

Table 3 Tumor location

Table 4 Histologic types of biopsy specimens

Table 5 Depth of tumor invasion, cT (UICC TNM 6th)

Table 6 Lymph node metastasis, cN (UICC TNM 6th)

Table 7 Distant metastasis, cM (UICC TNM 6th)

Table 8 Clinical stage (UICC TNM 6th)

II. Results of endoscopically treated patients in $\mathbf{2 0 0 9}$

Table 9 Details of endoscopic treatment

Table 10 Complications of EMR/ESD

Table 11 Pathological depth of tumor invasion of EMR/ESD specimens

Figure 1 Survival of patients treated with EMR/ ESD

Figure 2 Survival of patients treated with EMR/ ESD according to the pathological depth of tumor invasion (pT)

Figure 3 Survival of patients treated with EMR/ ESD according to the lymphatic and venous invasion

III. Results in patients treated with chemotherapy and/ or radiotherapy in 2009

Table 12 Dose of radiation (non-surgically treated cases)

Table 13 Dose of radiation (surgically treated cases)

Figure 4 Survival of patients treated with chemotherapy and/or radiotherapy (cStage I-IIA)

Figure 5 Survival of patients treated with chemotherapy and/or radiotherapy (cStage IIB-IVB)

IV. Results in patients underwent esophagectomy in 2009

Table 14 Treatment modalities of esophagectomy

Table 15 Tumor location
Table 16 Approaches to tumor resection

Table 17 Video-assisted surgery

Table 18 Fields of lymph node dissection according to the location of the tumor

Table 19 Reconstruction route

Table 20 Organs used for reconstruction

Table 21 Histological classification

Table 22 Depth of tumor invasion, pT (JES 10th)

Table 23 Pathological grading of lymph node metastasis, pN (JES 10th)

Table 24 Numbers of the metastatic nodes

Table 25 Pathological findings of distant organ metastasis, pM (JES 10th)

Table 26 Residual tumor, $\mathbf{R}$

Table 27 Causes of death

Figure 6 Survival of patients who underwent esophagectomy

Figure 7 Survival of patients who underwent esophagectomy according to clinical stage (JES TNM 10th)

Figure 8 Survival of patients who underwent esophagectomy according to clinical stage (UICC TNM 6th)

Figure 9 Survival of patients who underwent esophagectomy according to the depth of tumor invasion (JES 10th: pT)

Figure 10 Survival of patients who underwent esophagectomy according to the depth of tumor invasion (UICC TNM 6th: pT)

Figure 11 Survival of patients who underwent esophagectomy according to lymph node metastasis (JES 10th: pN)

Figure 12 Survival of patients who underwent esophagectomy according to lymph node metastasis (UICC TNM 6th: pN)

Figure 13 Survival of patients who underwent esophagectomy according to number of metastatic nodes

Figure 14 Survival of patients who underwent esophagectomy according to pathological stage (JES 10th)

Figure 15 Survival of patients who underwent esophagectomy according to pathological stage (UICC TNM 6th)

Figure 16 Survival of patients who underwent esophagectomy according to residual tumor $(\mathbf{R})$ 


\section{Clinical factors of esophageal cancer patients treated in 2009}

Institution-registered cases in 2009

Institutions

Aichi Cancer Center

Aizawa Hospital

Akita University Hospital

Aomori Municipal Hospital

Aomori Prefectural Central Hospital

Arao Municipal Hospital

Asahikawa Medical College Hospital

Chiba Cancer Center

Chiba Medical Center

Chiba University Hospital

Chibaken Saiseikai Narashino Hospital

Dokkyo Medical University Hospital

Ehime University Hospital

Foundation for Detection of Early Gastric Carcinoma

Fuchu Hospital

Fujioka General Hospital

Fujisawa Shounandai Hospital

Fujita Health University

Fukui Prefectural Hospital

Fukui University Hospital

Fukuoka Dental College and Dental Hospital

Fukuoka Saiseikai General Hospital?

Fukuoka University Hospital

Fukuoka Wajiro Hospital

Fukushima Medical University Hospital

Fukuyama City Hopital

Gifu Prefectural General Medical Center

Gifu University Hospital

Gunma Central General Hospital

Gunma Prefectural Cancer Center

Gunma University Hospital

Gunmaken Saiseikai Maebashi Hospital

Hachinohe City Hospital

Hakodate Goryokaku Hospital

Hakodate National Hospital

Hamamatsu University School of Medicine, University Hospital

Handa City Hospital

Hannan Chuo Hospital

Heartlife Hospital

Higashiosaka City General Hospital

Hino Memorial Hospital

Hiratsuka City Hospital

Hiratsuka Kyosai Hospital
Continued

Institutions

Hirosaki University Hospital

Hiroshima City Asa Hospital

Hiroshima University Research Institute for Radiation Biology Medicine

Hitachi General Hospital

Hofu Institute of Gastroenterology

Hokkaido Kin-Ikyo Chuo Hospital

Hokkaido University Hospital

Hyogo Cancer Center

Hyogo College of Medicine

Hyogo Prefectural Nishinomiya Hospital

Ibaraki Prefectural Central Hospital

Iizuka Hospital

Imazu Surgical Clinic

Inazawa City Hospital

Internatinal University of Health and Welfare Hospital

Isehara Kyodo Hospital

Ishikawa Prefectural Central Hospital

Iwakuni Medical Center

Iwate Medical University Hospital

Iwate Prefectural Chubu Hospital

Iwate Prefectural Isawa Hospital

Japanese Red Cross Fukui Hospital

Japanese Red Cross Ishinomaki Hospital

Japanese Red Cross Kyoto Daini Hospital?

Japanese Red Cross Maebashi Hospital

Japanese Red Cross Nagaoka Hospital

Japanese Red Cross Narita Hospital

Japanese Red Cross Nasu Hospital

Jichi Medical University Hospital

Juntendo University Hospital

Juntendo University Shizuoka Hospital

Junwakai Memorial Hospital

Kagawa Rosai Hospital

Kagawa University Hospital

Kagoshima Kenritsu Satsunan Hospital

Kagoshima University Hospital

Kameda General Hospital

Kanagawa Cancer Center

Kanazawa Medical University Hospital

Kanazawa University Hospital

Kansai Medical University Hirakata Hospital

Kansai Rosai Hospital

Kashiwa Kousei General Hospital

Kawakita General Hospital

Kawasaki Medical School Hospital

Kawasaki Medical School Kawasaki Hospital

Kawasaki Municipal Hospital

Kawasaki Municipal Ida Hospital 
Continued

Institutions

Keio University Hospital

Keiyukai Sapporo Hospital

Kikuna Memorial Hospital

Kinki Central Hospital

Kinki University Hospital

Kiryu Kosei General Hospital

Kishiwada City Hospital

Kitaakita Municipal Hospital

Kitakyushu Municipal Medical Center

Kitano Hospital

Kitasato University Hospital

Kobe City Medical Center General Hospital

Kobe University Hospital

Kochi University Hospital

Kokura Memorial Hospital

Kumamoto City Hospital

Kumamoto University Hospital

Kurashiki Central Hospital

Kurume General Hospital

Kurume University Hospital

Kuwana West Medical Center

Kyoto University Hospital

Kyushu Central Hospital of the Mutual Aid Association of Public School Teachers

Kyushu University Beppu Hospital

Kyushu University Hospital

Kyushu Medical Center

Machida Municipal Hospital

Matsuda Hospital

Matsushita Memorial Hospital

Matsuyama Red Cross Hospital

Mie University Hospital

Mino City Hospital

Mito Red Cross Hospital

Mitsui Memorial Hospital

Miyazaki Konan Hospital

Murakami General Hospital

Musashimurayama Hospital

Musashino Red Cross Hospital

Nagahama City Hospital

Nagano Red Cross Hospital

Nagaoka Chuo General Hospital

Nagasaki University Hospital

Nagayoshi General Hospital

Nagoya City University Hospital

Nagoya City West Medical Center

Nagoya Daiichi Red Cross Hospital

Nagoya University Hospital

Nara Hospital Kinki University Faculty of Medicine
Continued

Institutions

Nara Medical University Hospital

National Cancer Center Hospital

National Cancer Center Hospital East

National Center for Global Health and Medicine

National Defense Medical College Hospital

National Hospital Organization Beppu Medical Center

National Hospital Organization Chiba-East-Hospital

National Hospital Organization Fukuoka-higashi Medical Center

National Hospital Organization Kure Medical Center

National Hospital Organization Kyoto Medical Center

National Hospital Organization Kyushu Cancer Center

National Hospital Organization Matsumoto National Hospital

National Hospital Organization Nagasaki Medical Center.

National Hospital Organization Nagoya Medical Center

National Hospital Organization Osaka National Hospital

National Hospital Organization Tokyo Medical Center

Niigata Cancer Center Hospital

Niigata City General Hospital

Niigata Prefectural Shibata Hospital

Niigata University Medical and Dental Hospital

Nikko Memorial Hospital

Nippon Medical School Chiba Hokusoh Hospital

Nippon Medical School Hospital

Nippon Medical School Musashi Kosugi Hospital

Nippon Medical School Tama Nagayama Hospital

Nishi-Kobe Medical Center

Nishinomiya Municipal Central Hospital

Numazu City Hospital

Obihiro Kousei General Hospital

Ohta General Hospital Foundation Ohta Nishinouchi Hospital

Oita Red Cross Hospital

Oita University Hospital

Okayama Saiseikai General Hospital

Okayama University Hospital

Onomichi Municipal Hospital

Osaka City General Hospital

Osaka City University Hospital

Osaka Hospital of Japan Seafarers relief Association

Osaka Medical Center for Cancer and Cardiovascular Diseases

Osaka Medical College Hospital

Osaka Police Hospital

Osaka Prefectural Hospital Organization Osaka General Medical Center

Osaka Red Cross Hospital

Osaka University Hospital

Otsu Municipal Hospital

Otsu Red Cross Hospital

Rakusei Hospital

Ryukyu University Hospital 
Continued

\section{Institutions}

Saga University Hospital

Saga-ken Medical Center Koseikan

Saiseikai Fukushima General Hospital

Saiseikai Hiroshima Hospital

Saiseikai Kyoto Hospital

Saiseikai Utsunomiya Hospital

Saitama City Hospital

Saitama Medical Center Jichi Medical University

Saitama Medical University Hospital

Saitama Medical University Saitama International Medical Center

Saitama Medical University Saitama Medical Center

Saitama Medical Center

Sakai City Medical Center

Saku Central Hospital

Sanin Rosai Hospital

Sano Kousei General Hospital

Sendai City Hospital

Shiga Medical Center for Adults

Shiga University of Medical Science Hospital

Shikoku Cancer Center

Shimada Hospital

Shimane University Hospital

Shimizu Welfare Hospital

Shinshu University Hospital

Shizuoka Cancer Center

Shizuoka City Shizuoka Hospital

Shizuoka General Hospital

Showa University Hospital

Showa University Northern Yokohama Hospital

Showa University Koto-Toyosu Hospital

Social Insurance Omuta Tenryo Hospital

Social Insurance Tagawa Hospital

Yokohama Chuo Hospital

Sonoda Daiichi Hospital

St. Marianna University School of Medical Hospital

St. Luke's International Hospital

Sugita Genpaku Memorial Obama Municipal Hospital

Suita Municipal Hospital

Takasago Municipal Hospital

Takatsuki Red Cross Hospital

Teikyo University Hospital

Tenri Hospital

The Cancer Institute Hospital of JFCR

The Jikei University Hospital

The Research Center Hospital for Charged Particle Therapy of NIRS

Toho University Omori Medical Center
Continued

Institutions

Toho University Sakura Medical Center

Tohoku Kosai Hospital

Tohoku University Hospital

Tokai University Hachioji Hospital

Tokai University Hospital

Tokai University Tokyo Hospital

Tokushima Municipal Hospital

Tokushima Red Cross Hospital

Tokushima University Hospital

Tokyo Dental College Ichikawa General Hospital

Tokyo Medical and Dental University Hospital

Tokyo Medical University Hospital

Tokyo Medical University Ibaraki Medical Center

Tokyo Metropolitan Cancer and Infectious Center Komagome Hospital

Tokyo Metropolitan Health and Medical Corporation Toshima Hospital

Tokyo University Hospital

Tokyo Women's Medical University Hospital

Tokyo Women's Medical University Medical Center East

Tonan Hospital

Tone Chuou Hospital

Tottori Prefectural Central Hospital

Tottori University Hospital

Toyama Prefectural Central Hospital

Toyama University Hospital

Tsuchiura Kyodo Hospital

Tsukuba University Hospital

Tsuruoka Municipal Shonai Hospital

"University Hospital, Kyoto Prefectural University of Medicine"

University of Miyazaki Hospital

Wakayama Medical University Hospital

Yamagata Prefectural and Sakata Municipal Hospital Organization

Yamagata Prefectural Central Hospital

Yamagata Prefectural Shinjo Hospital

Yamagata University Hospital

Yamaguchi University Hospital

Yamaguchi-ken Saiseikai Shimonoseki General Hospital

Yamanashi Prefectural Central Hospital

Yamanashi University Hospital

Yao Municipal Hospital

Yokohama City Municipal Hospital

Yokohama City University Hospital

Yokohama City University Medical Center

Yuri General Hospital

(Total 276 institutions) 


\section{Patient Background}

Table 1 Age and gender

\begin{tabular}{lrrlr}
\hline Age & Male & Female & Unknown & \multicolumn{1}{c}{ Cases $(\%)$} \\
\hline$\sim 29$ & 6 & 1 & 0 & $7(0.1 \%)$ \\
$30-39$ & 12 & 6 & 0 & $18(0.3 \%)$ \\
$40-49$ & 121 & 34 & 0 & $155(2.5 \%)$ \\
$50-59$ & 946 & 173 & 1 & $1120(17.9 \%)$ \\
$60-69$ & 2332 & 354 & 0 & $2686(42.9 \%)$ \\
$70-79$ & 1575 & 270 & 1 & $1846(29.5 \%)$ \\
$80-89$ & 303 & 75 & 0 & $378(6.0 \%)$ \\
$90 \sim$ & 16 & 3 & 0 & $19(0.3 \%)$ \\
Unknown & 27 & 4 & 0 & $31(0.5 \%)$ \\
Total & 5338 & 920 & 2 & $6260(100 \%)$ \\
\hline
\end{tabular}

Table 2 Primary treatment

\begin{tabular}{lc}
\hline Treatments & Cases $(\%)$ \\
\hline Surgery & $3943(63.0 \%)$ \\
Esophagectomy & $3844(61.8 \%)$ \\
Palliative & $99(1.2 \%)$ \\
Chemotherapy/radiotherapy & $1383(22.1 \%)$ \\
Endoscopic treatment & $932(14.9 \%)$ \\
Others & $2(0.0 \%)$ \\
Total & $6260(100 \%)$ \\
\hline
\end{tabular}

Table 3 Tumor location

\begin{tabular}{|c|c|c|c|c|c|c|}
\hline Location of tumor & $\begin{array}{l}\text { Endoscopic } \\
\text { treatment }(\%)\end{array}$ & $\begin{array}{l}\text { Chemotherapy and/or } \\
\text { radiotherapy }(\%)\end{array}$ & Palliative surgery (\%) & Esophagectomy (\%) & Other $(\%)$ & Total (\%) \\
\hline Cervical & $18(1.9 \%)$ & $112(8.1 \%)$ & $9(9.1 \%)$ & $137(3.6 \%)$ & 0 & $276(4.4 \%)$ \\
\hline Upper thoracic & $105(11.3 \%)$ & $184(13.3 \%)$ & $19(19.2 \%)$ & $437(11.4 \%)$ & 0 & $745(11.9 \%)$ \\
\hline Middle thoracic & $511(54.8 \%)$ & $665(48.1 \%)$ & $50(50.5 \%)$ & $1778(46.3 \%)$ & $1(50.0 \%)$ & $3005(48.0 \%)$ \\
\hline Lower thoracic & $243(26.1 \%)$ & $325(23.5 \%)$ & $18(18.2 \%)$ & $1147(29.8 \%)$ & 0 & $1733(27.7 \%)$ \\
\hline $\mathrm{E}>\mathrm{G}$ & $40(4.3 \%)$ & $38(2.7 \%)$ & $2(2.0 \%)$ & $245(6.4 \%)$ & 0 & $325(5.2 \%)$ \\
\hline $\mathrm{E}=\mathrm{G}$ & $5(0.5 \%)$ & $6(0.4 \%)$ & 0 & $41(1.1 \%)$ & 0 & $52(0.8 \%)$ \\
\hline $\mathrm{G}>\mathrm{E}$ & 0 & $2(0.1 \%)$ & 0 & $34(0.9 \%)$ & 0 & $36(0.6 \%)$ \\
\hline Unknown & $10(1.1 \%)$ & $51(3.7 \%)$ & $1(1.0 \%)$ & $25(0.7 \%)$ & $1(50.0 \%)$ & $88(1.4 \%)$ \\
\hline Total & $932(100 \%)$ & $1383(100 \%)$ & $99(100 \%)$ & $3844(100 \%)$ & $2(100 \%)$ & $6260(100 \%)$ \\
\hline
\end{tabular}

$E$ esophageal, $G$ gastric 
Table 4 Histologic types of biopsy specimens

\begin{tabular}{lc}
\hline Histologic types & Cases $(\%)$ \\
\hline Squamous cell carcinoma & $5665(90.5 \%)$ \\
Squamous cell carcinoma & $3827(61.1 \%)$ \\
Well differentiated & $354(5.7 \%)$ \\
Moderately differentiated & $1140(18.2 \%)$ \\
Poorly differentiated & $344(5.5 \%)$ \\
Adenocarcinoma & $296(4.7 \%)$ \\
Adenosquamous carcinoma & $13(0.2 \%)$ \\
Mucoepidermoid carcinoma & $1(0.0 \%)$ \\
Basaloid carcinoma & $22(0.4 \%)$ \\
Neuroendocrine cell carcinoma & $14(0.2 \%)$ \\
Undifferentiated carcinoma & $10(0.2 \%)$ \\
Malignant melanoma & $7(0.1 \%)$ \\
Carcinosarcoma & $17(0.3 \%)$ \\
Other tumors & $28(0.4 \%)$ \\
Unknown & $187(3.0 \%)$ \\
Total & $6260(100 \%)$ \\
\hline
\end{tabular}

\begin{tabular}{lc}
\hline cT & Cases $(\%)$ \\
\hline cTX & $29(0.5 \%)$ \\
cT0 & $11(0.2 \%)$ \\
cTis & $157(2.5 \%)$ \\
cT1 & $359(5.7 \%)$ \\
cT1a & $650(10.4 \%)$ \\
cT1b & $1134(18.1 \%)$ \\
cT2 & $868(13.9 \%)$ \\
cT3 & $2252(36.0 \%)$ \\
cT4 & $701(11.2 \%)$ \\
Unknown & $99(1.6 \%)$ \\
Total & $6260(100 \%)$ \\
\hline
\end{tabular}

Table 5 Depth of tumor invasion, cT (UICC TNM 6th)
Table 6 Lymph node metastasis, cN (UICC TNM 6th)

\begin{tabular}{lc}
\hline $\mathrm{cN}$ & Cases $(\%)$ \\
\hline $\mathrm{cNX}$ & $72(1.2 \%)$ \\
$\mathrm{cN} 0$ & $2920(46.6 \%)$ \\
$\mathrm{cN} 1$ & $3157(50.4 \%)$ \\
Unknown & $111(1.8 \%)$ \\
Total & $6260(100 \%)$ \\
\hline
\end{tabular}

Table 7 Distant metastasis, cM (UICC TNM 6th)

\begin{tabular}{lc}
\hline $\mathrm{cM}$ & Cases $(\%)$ \\
\hline $\mathrm{cMX}$ & $57(0.9 \%)$ \\
$\mathrm{cM} 0$ & $5295(84.6 \%)$ \\
$\mathrm{cM} 1$ & $223(3.6 \%)$ \\
$\mathrm{cM} 1 \mathrm{a}$ & $141(2.3 \%)$ \\
$\mathrm{cM} 1 \mathrm{~b}$ & $466(7.4 \%)$ \\
Total & $6260(100 \%)$ \\
\hline
\end{tabular}


Table 8 Clinical Stage (UICC TNM 6th)

Location of tumor Endoscopic treatment (\%)Chemotherapy and/or $\quad$ Palliative surgery (\%) Esophagectomy (\%) Other (\%) Total (\%) radiotherapy $(\%)$

\begin{tabular}{|c|c|c|c|c|c|c|}
\hline 0 & $131(14.1 \%)$ & $6(0.4 \%)$ & $1(1.0 \%)$ & $13(0.3 \%)$ & 0 & $151(2.4 \%)$ \\
\hline I & $658(70.6 \%)$ & $152(11.0 \%)$ & $2(2.0 \%)$ & $964(25.1 \%)$ & 0 & $1776(28.4 \%)$ \\
\hline IIA & $6(0.6 \%)$ & $125(9.0 \%)$ & $7(7.1 \%)$ & $717(18.7 \%)$ & 0 & $855(13.7 \%)$ \\
\hline IIB & $7(0.8 \%)$ & $98(7.1 \%)$ & $2(2.0 \%)$ & $555(14.4 \%)$ & 0 & $662(10.6 \%)$ \\
\hline III & $29(3.1 \%)$ & $452(32.7 \%)$ & $62(62.6 \%)$ & $1243(32.3 \%)$ & $1(50.0 \%)$ & $1787(28.5 \%)$ \\
\hline IV & $10(1.1 \%)$ & $139(10.1 \%)$ & $7(7.1 \%)$ & $44(1.1 \%)$ & 0 & $200(3.2 \%)$ \\
\hline IVA & $5(0.5 \%)$ & $53(3.8 \%)$ & $1(1.0 \%)$ & $81(2.1 \%)$ & 0 & $140(2.2 \%)$ \\
\hline IVB & $18(1.9 \%)$ & $265(19.2 \%)$ & $12(12.1 \%)$ & $156(4.1 \%)$ & 0 & $451(7.2 \%)$ \\
\hline Unknown & $68(7.3 \%)$ & $93(6.7 \%)$ & $5(5.1 \%)$ & $71(1.8 \%)$ & $1(50.0 \%)$ & $238(3.8 \%)$ \\
\hline Total & $932(100 \%)$ & $1383(100 \%)$ & $99(100 \%)$ & $3844(100 \%)$ & $2(100 \%)$ & $6260(100 \%)$ \\
\hline
\end{tabular}

\section{Results of endoscopically treated patients in 2009}

Table 9 Details of endoscopic treatment

\begin{tabular}{lc}
\hline Treatment details & Cases $(\%)$ \\
\hline EMR & $201(21.6 \%)$ \\
EMR + ESD & $11(1.2 \%)$ \\
EMR + YAG laser & $7(0.8 \%)$ \\
ESD & $607(65.1 \%)$ \\
ESD + other treatment & $7(0.8 \%)$ \\
PDT & $2(0.2 \%)$ \\
PDT + YAG laser & $2(0.2 \%)$ \\
YAG laser & $10(1.1 \%)$ \\
Esophageal stenting & $70(7.5 \%)$ \\
Esophageal stenting + tracheal stenting & $2(0.2 \%)$ \\
Tracheal stenting & $4(0.4 \%)$ \\
Others & $5(0.5 \%)$ \\
Unknown & $4(0.4 \%)$ \\
Total & $753(100 \%)$ \\
\hline EMR endoscopic mucosal resection, ESD endoscopic & submucosal \\
dissection, YAG: yttrium aluminum garnet, PDT photodynamic ther- & \\
apy & \\
Table 10 Complications of EMR/ESD & \\
\hline Complications of EMR/ESD & \\
\hline None & \\
Perforation & \\
Bleeding & $166(91.8 \%)$ \\
Mediastinitis & $16(1.9 \%)$ \\
Stenosis & $2(0.2 \%)$ \\
Others & 0 \\
\hline
\end{tabular}

Table 11 Pathological depth of tumor invasion of EMR/ESD specimens

\begin{tabular}{ll}
\hline Pathological depth of tumor invasion & Cases $(\%)$ \\
\hline pTX & $1(0.1 \%)$ \\
pT0 & $5(0.6 \%)$ \\
pTis & $166(19.9 \%)$ \\
pT1a & $507(60.9 \%)$ \\
pT1b & $86(10.3 \%)$ \\
pT2 & 0 \\
Unknown & $68(8.2 \%)$ \\
Total & $833(100 \%)$ \\
\hline
\end{tabular}


Fig. 1 Survival of patients treated with EMR/ESD

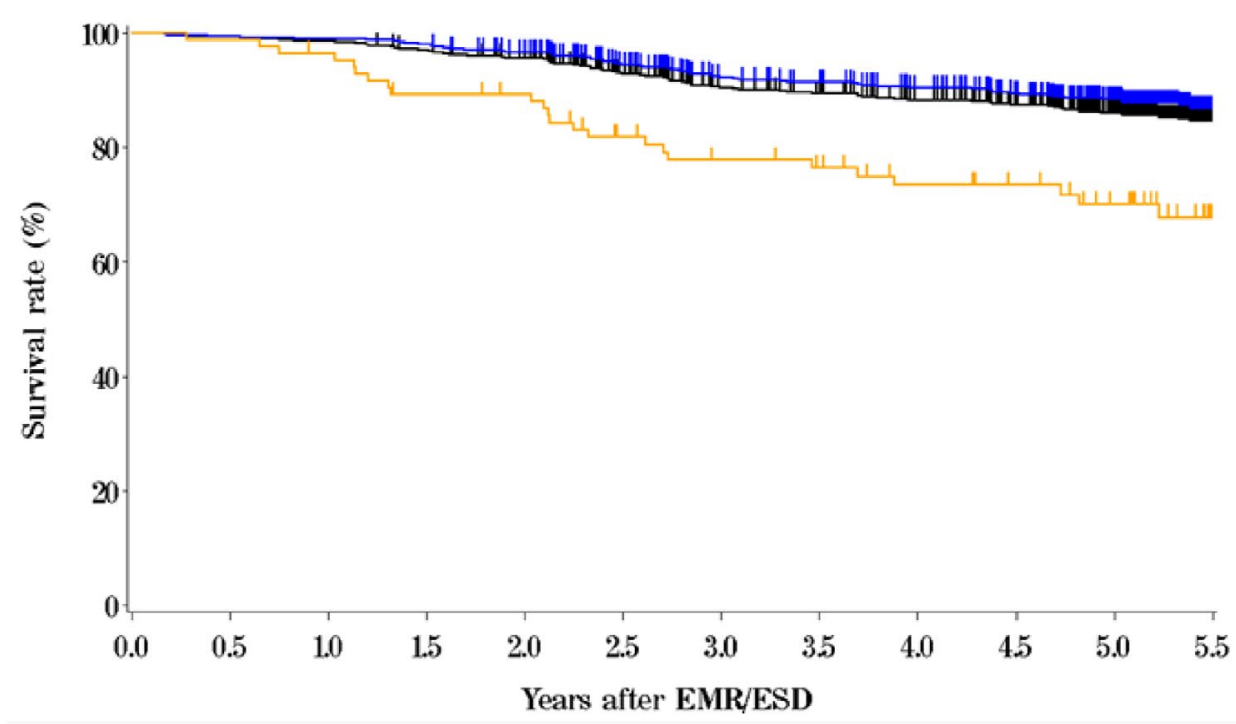

Total $(n=725)$

Complete resection $(n=638)$

Incomplete resection $(n=87)$

\begin{tabular}{lccccc}
\hline & \multicolumn{5}{c}{ Years after EMR/ESD } \\
\cline { 2 - 6 } & 1 & 2 & 3 & 4 & 5 \\
\hline Total & $98.7 \%$ & $95.7 \%$ & $90.4 \%$ & $88.3 \%$ & $86.2 \%$ \\
Complete resection & $99.0 \%$ & $96.7 \%$ & $92.2 \%$ & $90.4 \%$ & $88.4 \%$ \\
Incomplete resection & $\mathbf{9 6 . 5 \%}$ & $\mathbf{8 9 . 3 \%}$ & $\mathbf{7 7 . 8 \%}$ & $\mathbf{7 3 . 4 \%}$ & $\mathbf{7 0 . 1 \%}$ \\
\hline
\end{tabular}


Fig. 2 Survival of patients treated with EMR/ESD according to the pathological depth of tumor invasion (pT)

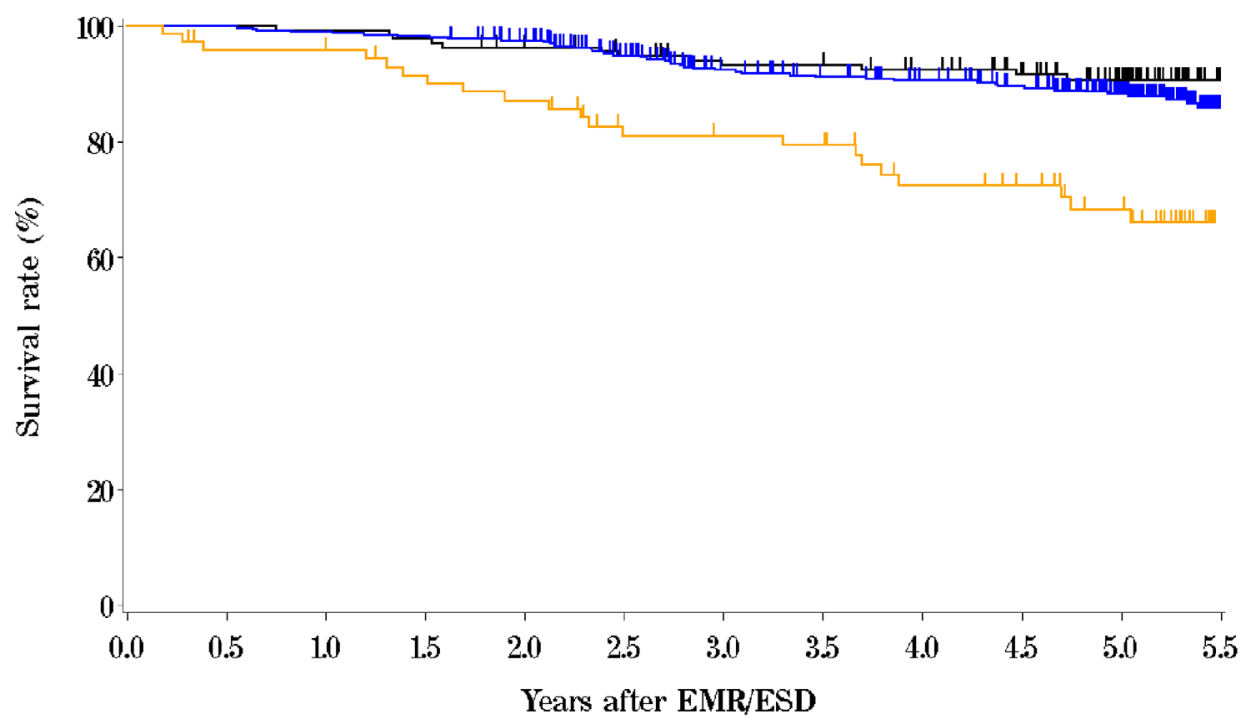

pTis $(n=146) \quad$ pT1a $(n=441) \quad$ pT1b $(n=75)$

\begin{tabular}{lccccc}
\hline & \multicolumn{5}{c}{ Years after EMR/ESD } \\
\cline { 2 - 6 } & 1 & 2 & 3 & 4 & 5 \\
\hline pTis & $99.3 \%$ & $96.4 \%$ & $93.3 \%$ & $92.5 \%$ & $90.7 \%$ \\
pT1a & $99.0 \%$ & $97.6 \%$ & $92.4 \%$ & $90.6 \%$ & $88.2 \%$ \\
pT1b & $95.9 \%$ & $87.2 \%$ & $81.1 \%$ & $72.5 \%$ & $68.3 \%$ \\
\hline
\end{tabular}


Fig. 3 Survival of patients treated with EMR/ESD according to the lymphatic and venous invasion

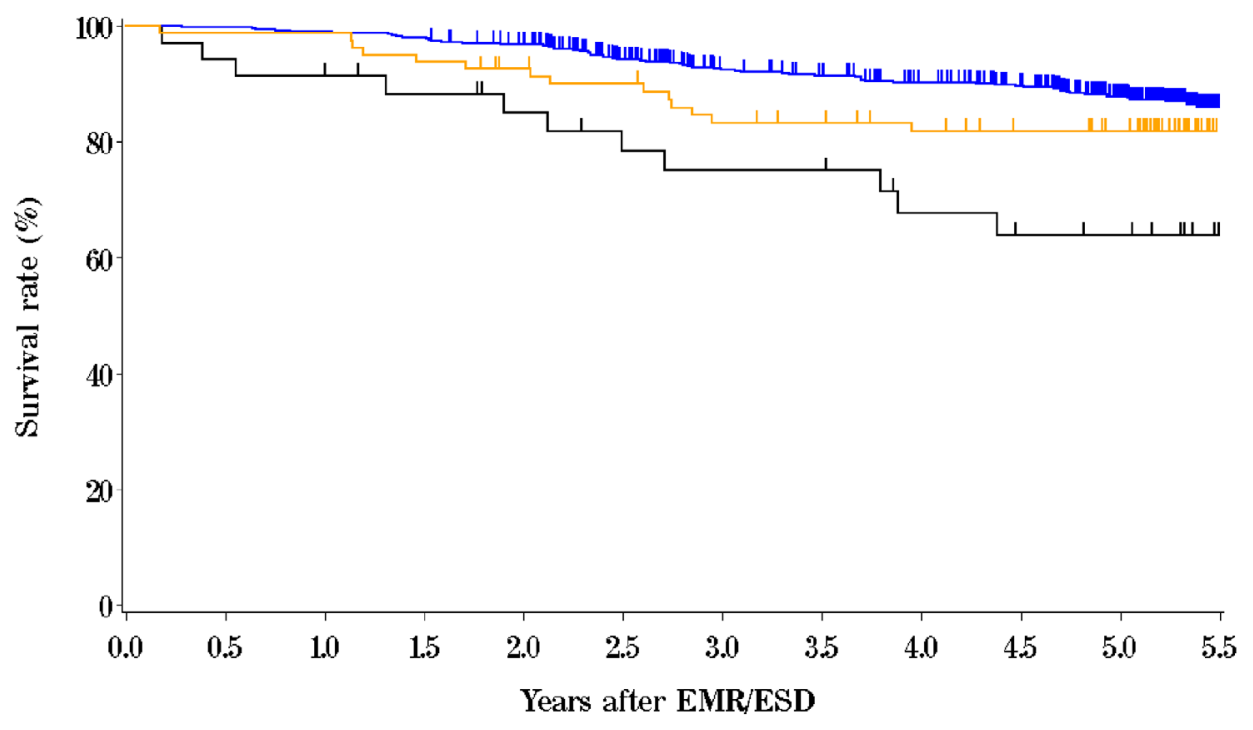

ly0 and v0 $(n=612)$
Unknown $(n=84)$

\begin{tabular}{lccccc}
\hline & \multicolumn{5}{c}{ Years after EMR/ESD } \\
\cline { 2 - 6 } & 1 & 2 & 3 & 4 & 5 \\
\hline ly0 and v0 & $99.1 \%$ & $96.8 \%$ & $92.4 \%$ & $90.4 \%$ & $88.0 \%$ \\
ly1-3 or v1-3 & $91.4 \%$ & $85.1 \%$ & $75.0 \%$ & $67.7 \%$ & $63.9 \%$ \\
Unknown & $98.8 \%$ & $92.6 \%$ & $83.3 \%$ & $81.8 \%$ & $81.8 \%$ \\
\hline
\end{tabular}




\section{Results in patients treated with chemotherapy and/or radiotherapy in $\mathbf{2 0 0 9}$}

Table 12 Dose of radiation (non-surgically treated cases)

\begin{tabular}{|c|c|c|c|c|c|c|c|}
\hline \multirow{2}{*}{$\begin{array}{l}\text { Dose of radiation } \\
(\mathrm{Gy})\end{array}$} & \multicolumn{2}{|l|}{ Definitive } & \multirow[t]{2}{*}{ Palliative (\%) } & \multirow[t]{2}{*}{ Recurrence (\%) } & \multirow[t]{2}{*}{ Others $(\%)$} & \multirow[t]{2}{*}{ Unknown (\%) } & \multirow[t]{2}{*}{ Total (\%) } \\
\hline & $\begin{array}{l}\text { Radiation alone } \\
(\%)\end{array}$ & $\begin{array}{l}\text { With chemother- } \\
\text { apy }(\%)\end{array}$ & & & & & \\
\hline-29 & $5(4.1 \%)$ & $18(2.3 \%)$ & $23(11.0 \%)$ & $2(6.1 \%)$ & 0 & $1(5.6 \%)$ & $49(4.1 \%)$ \\
\hline $30-39$ & $1(0.8 \%)$ & $15(1.9 \%)$ & $25(11.9 \%)$ & $3(9.1 \%)$ & $1(7.1 \%)$ & 0 & $45(3.8 \%)$ \\
\hline $40-49$ & $11(8.9 \%)$ & $40(5.1 \%)$ & $31(14.8 \%)$ & $9(27.3 \%)$ & $8(57.1 \%)$ & $1(5.6 \%)$ & $100(8.4 \%)$ \\
\hline $50-59$ & $24(19.5 \%)$ & $199(25.3 \%)$ & $47(22.4 \%)$ & $8(24.2 \%)$ & $2(14.3 \%)$ & $1(5.6 \%)$ & $281(23.7 \%)$ \\
\hline $60-69$ & $74(60.2 \%)$ & $493(62.6 \%)$ & $81(38.6 \%)$ & $9(27.3 \%)$ & $2(14.3 \%)$ & $15(83.3 \%)$ & $674(56.8 \%)$ \\
\hline $70-$ & $6(7.2 \%)$ & $8(2.1 \%)$ & $2(0.0 \%)$ & 0 & 0 & 0 & $16(2.2 \%)$ \\
\hline Unknown & $2(1.6 \%)$ & $15(1.9 \%)$ & $1(0.5 \%)$ & $2(6.1 \%)$ & $1(7.1 \%)$ & 0 & $21(1.8 \%)$ \\
\hline Total & $123(100 \%)$ & $788(100 \%)$ & $210(100 \%)$ & $33(100 \%)$ & $14(100 \%)$ & $18(100 \%)$ & $1186(100 \%)$ \\
\hline $\begin{array}{l}\text { Median } \\
\quad(\min -\max )\end{array}$ & $60.0(6.0-120.0)$ & $60.0(2.0-124.0)$ & $54.0(2.0-95.4)$ & $50.0(20.0-66.0)$ & $40.0(36.0-60.0)$ & $60.0(2.0-61.2)$ & $60.0(2.0-124.0)$ \\
\hline
\end{tabular}

Table 13 Dose of radiation (surgically treated cases)

\begin{tabular}{llc}
\hline Dose of radiation (Gy) & $\begin{array}{l}\text { Preoperative radiation } \\
(\%)\end{array}$ & $\begin{array}{l}\text { Postoperative radia- } \\
\text { tion }(\%)\end{array}$ \\
\hline-29 & $3(1.4 \%)$ & $1(1.4 \%)$ \\
$30-39$ & $54(24.4 \%)$ & $2(2.7 \%)$ \\
$40-49$ & $132(59.7 \%)$ & $21(28.4 \%)$ \\
$50-59$ & $9(4.1 \%)$ & $18(24.3 \%)$ \\
$60-69$ & $15(6.8 \%)$ & $27(36.5 \%)$ \\
$70-$ & 0 & $0(1.1 \%)$ \\
Unknown & $8(3.6 \%)$ & $5(6.8 \%)$ \\
Total & $221(100 \%)$ & $74(100 \%)$ \\
Median (min-max) & $40.0(15.0-66.0)$ & $50.4(4.0-64.0)$
\end{tabular}


Fig. 4 Survival of patients treated with chemotherapy and/ or radiotherapy (cStage I-IIA)

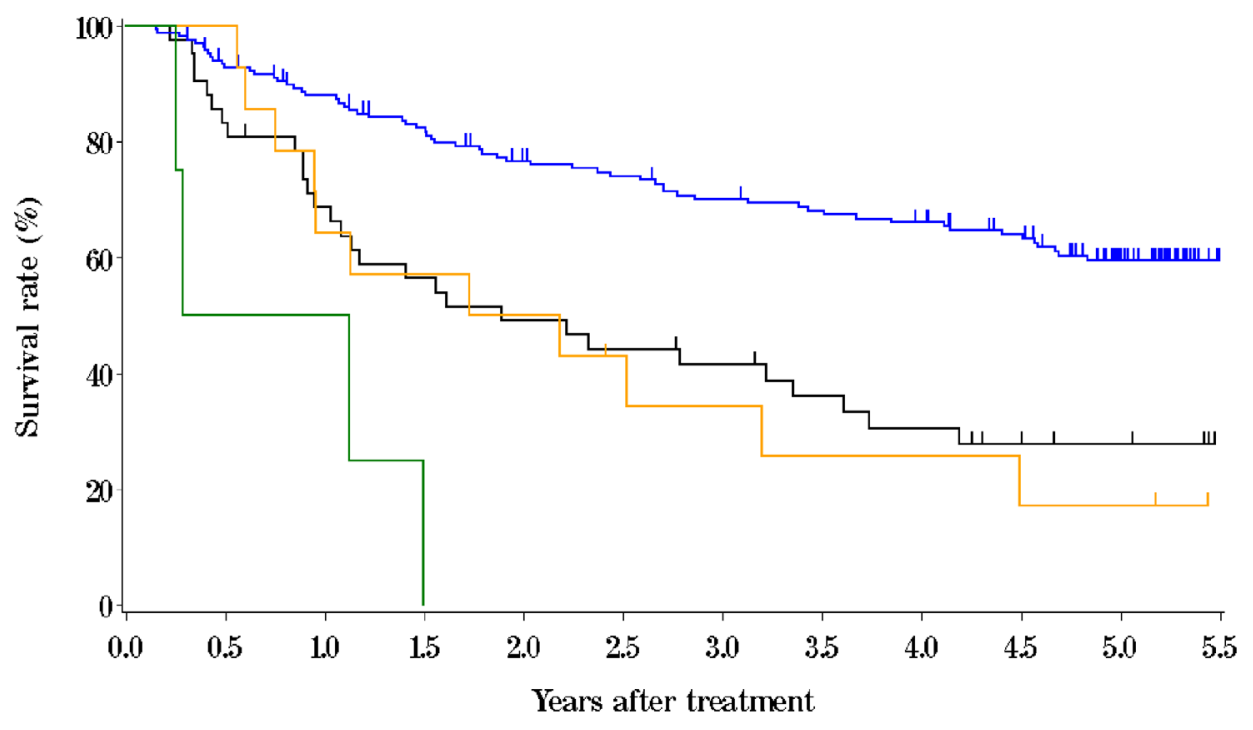

Chemoradiotherapy $(n=171)$

Radiotherapy alone $(n=42)$

Chemotherapy alone $(n=14)$

\begin{tabular}{lccccc}
\hline & \multicolumn{5}{c}{ Years after treatment } \\
\cline { 2 - 6 } & 1 & 2 & 3 & 4 & 5 \\
\hline Che moradiothe rapy & $\mathbf{8 8 . 0} \%$ & $76.7 \%$ & $70.8 \%$ & $66.1 \%$ & $59.5 \%$ \\
Radiothe rapy alone & $73.6 \%$ & $49.1 \%$ & $41.6 \%$ & $30.5 \%$ & $27.8 \%$ \\
Che mothe rapy alone & $\mathbf{6 4 . 3 \%}$ & $\mathbf{5 0 . 0} \%$ & $34.3 \%$ & $\mathbf{2 5 . 7} \%$ & $\mathbf{1 7 . 1} \%$ \\
Palliative radiothe rapy & $\mathbf{5 0 . 0} \%$ & $\mathbf{0 . 0} \%$ & - & - & - \\
\hline
\end{tabular}


Fig. 5 Survival of patients treated with chemotherapy and/or radiotherapy (cStage IIB-IVB)

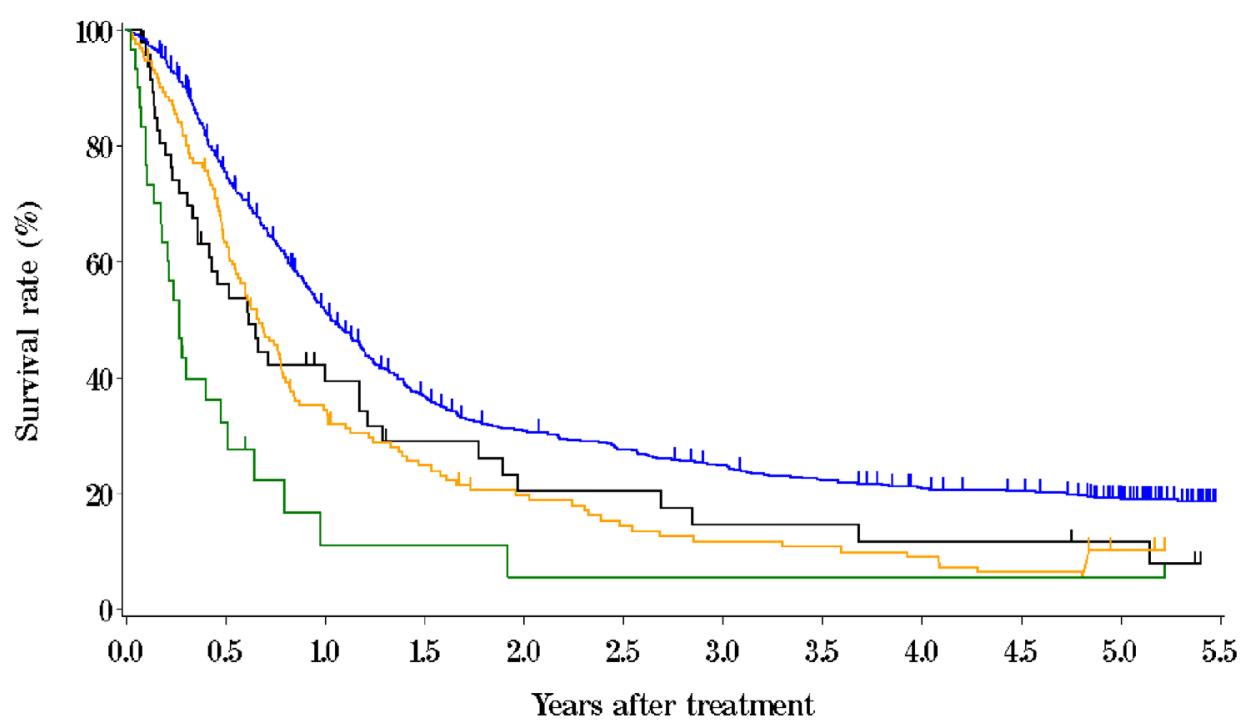

Chemoradiotherapy $(n=625)$

Radiotherapy alone $(n=48)$

Chemotherapy alone $(n=133)$

\begin{tabular}{lccccc}
\hline & \multicolumn{5}{c}{ Years after treatment } \\
\cline { 2 - 6 } & 1 & 2 & 3 & 4 & 5 \\
\hline Che moradiotherapy & $51.5 \%$ & $30.7 \%$ & $24.7 \%$ & $20.8 \%$ & $19.1 \%$ \\
Radiothe rapy alone & $39.4 \%$ & $20.2 \%$ & $14.4 \%$ & $11.6 \%$ & $11.6 \%$ \\
Che mothe rapy alone & $34.4 \%$ & $19.6 \%$ & $11.6 \%$ & $8.9 \%$ & $5.3 \%$ \\
Palliative radiothe rapy & $11.0 \%$ & $5.5 \%$ & $5.5 \%$ & $5.5 \%$ & $5.5 \%$ \\
\hline
\end{tabular}




\section{Results in patients who underwent esophagectomy in 2009}

Table 14 Treatment modalities of esophagectomy

\begin{tabular}{lc}
\hline Treatments & Cases $(\%)$ \\
\hline Esophagectomy & $1630(42.4 \%)$ \\
Esophagectomy + radiotherapy & $65(1.7 \%)$ \\
Esophagectomy + chemoradiotherapy & $655(17.0 \%)$ \\
$\begin{array}{l}\text { Esophagectomy + chemoradiotherapy + endoscopic } \\
\text { treatment }\end{array}$ & $16(0.4 \%)$ \\
$\begin{array}{l}\text { Esophagectomy + chemoradiotherapy + other treat- } \\
\text { ment }\end{array}$ & $2(0.1 \%)$ \\
$\begin{array}{l}\text { Esophagectomy + radiotherapy + endoscopic treat- } \\
\text { ment }\end{array}$ & $3(0.1 \%)$ \\
$\begin{array}{l}\text { Esophagectomy + radiotherapy + other treatment } \\
\text { Esophagectomy + chemotherapy }\end{array}$ & $1385(36.0 \%)$ \\
$\begin{array}{l}\text { Esophagectomy + chemotherapy + endoscopic treat- } \\
\text { ment }\end{array}$ & $8(0.2 \%)$ \\
Esophagectomy + chemotherapy + other treatment & $2(0.1 \%)$ \\
$\begin{array}{l}\text { Esophagectomy + endoscopic treatment } \\
\text { Total }\end{array}$ & $77(2.0 \%)$ \\
\hline
\end{tabular}

Table 15 Tumor location

\begin{tabular}{lc}
\hline Locations & Cases $(\%)$ \\
\hline Cervical & $137(3.6 \%)$ \\
Upper thoracic & $437(11.4 \%)$ \\
Middle thoracic & $1778(46.3 \%)$ \\
Lower thoracic & $1147(29.8 \%)$ \\
$\mathrm{E}>\mathrm{G}$ & $245(6.4 \%)$ \\
$\mathrm{E}=\mathrm{G}$ & $41(1.1 \%)$ \\
$\mathrm{G}>\mathrm{E}$ & $34(0.9 \%)$ \\
Unknown & $25(0.7 \%)$ \\
Total lesions & $3844(100 \%)$ \\
\hline
\end{tabular}

Table 16 Approaches to tumor resection

\begin{tabular}{lc}
\hline Approaches & Cases $(\%)$ \\
\hline Cervical approach & $132(3.4 \%)$ \\
Right thoracotomy & $3239(84.3 \%)$ \\
Left thoracotomy & $66(1.7 \%)$ \\
Left thoracoabdominal approach & $49(1.3 \%)$ \\
Laparotomy & $148(3.9 \%)$ \\
Transhiatal thoracic esophagectomy & $52(1.4 \%)$ \\
Transhiatal lower esophagectomy & $92(2.4 \%)$ \\
Sternotomy & $2(0.1 \%)$ \\
Others & $32(0.8 \%)$ \\
Unknown & $32(0.8 \%)$ \\
Total & $3844(100 \%)$ \\
\hline
\end{tabular}

Table 17 Video-assisted surgery

\begin{tabular}{lc}
\hline Video-assisted surgery & Cases $(\%)$ \\
\hline None & $2549(66.3 \%)$ \\
Thoracoscopy & $554(14.4 \%)$ \\
Laparoscopy & $124(3.2 \%)$ \\
Thoracoscopy + laparoscopy & $388(10.1 \%)$ \\
Mediastinoscopy & $26(0.7 \%)$ \\
Thoracoscopy + laparoscopy + mediastinoscopy & $4(0.1 \%)$ \\
Thoracoscopy + other & $11(0.3 \%)$ \\
Laparoscopy + mediastinoscopy & $5(0.1 \%)$ \\
Others & $17(0.4 \%)$ \\
Unknown & $166(4.3 \%)$ \\
Total & $3844(100 \%)$ \\
\hline
\end{tabular}




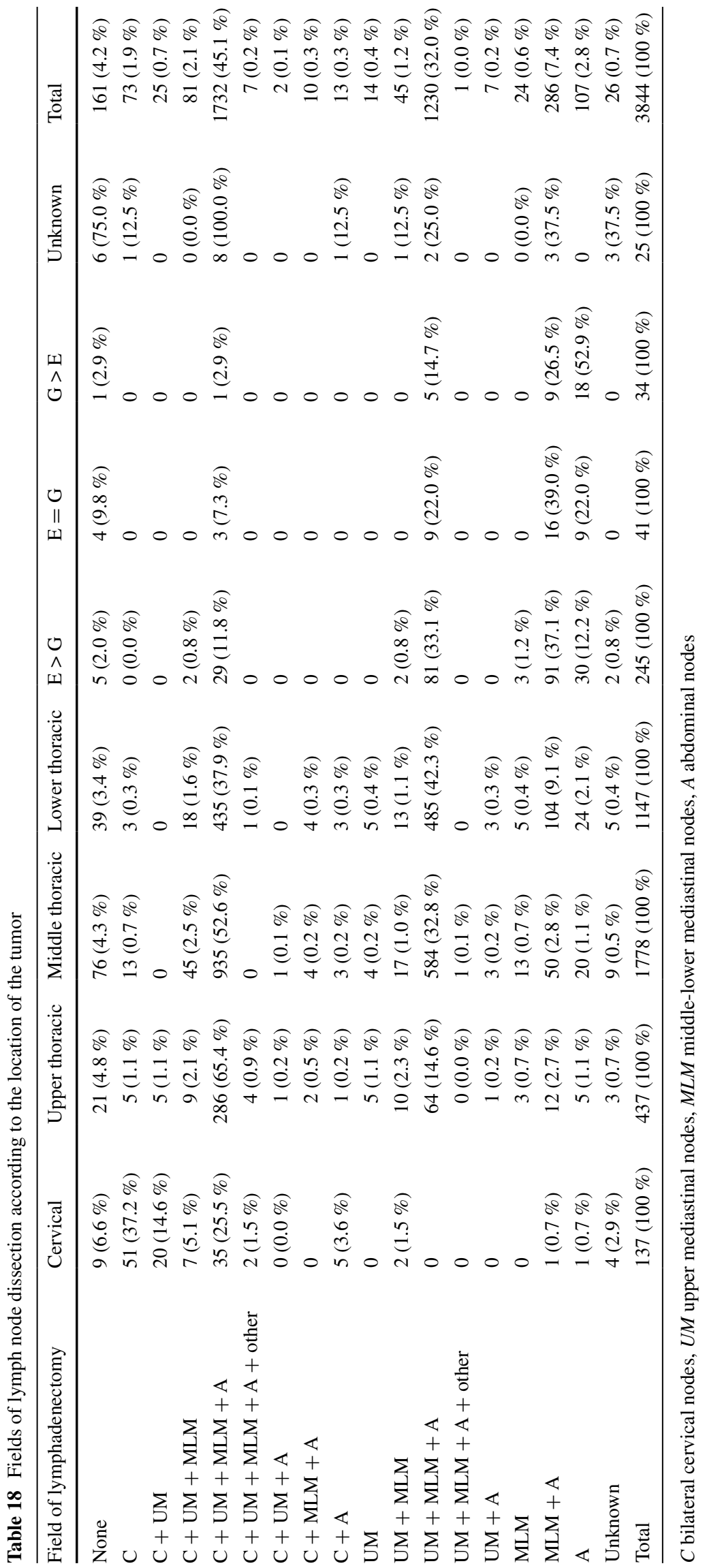


Table 19 Reconstruction route

\begin{tabular}{lc}
\hline Reconstruction route & Cases $(\%)$ \\
\hline None & $48(1.2 \%)$ \\
Subcutaneous & $323(8.4 \%)$ \\
Retrosternal & $1422(37.0 \%)$ \\
Intrathoracic & $446(11.6 \%)$ \\
Posterior mediastinal & $1491(38.8 \%)$ \\
Cervical & $49(1.3 \%)$ \\
Others & $36(0.9 \%)$ \\
Unknown & $29(0.8 \%)$ \\
Total & $3844(100 \%)$ \\
\hline
\end{tabular}

Table 20 Organs used for reconstruction

\begin{tabular}{lc}
\hline Organs used for reconstruction & Cases $(\%)$ \\
\hline None & $51(1.3 \%)$ \\
Whole stomach & $102(2.6 \%)$ \\
Gastric tube & $3234(81.6 \%)$ \\
Jejunum & $213(5.4 \%)$ \\
Free jejunum & $88(2.2 \%)$ \\
Colon & $153(3.9 \%)$ \\
Free colon & $12(0.3 \%)$ \\
Skin graft & $0(0.0 \%)$ \\
Others & $93(2.3 \%)$ \\
Unknown & $18(0.5 \%)$ \\
Total organs & $3964(100 \%)$ \\
Total cases & 3844 \\
\hline
\end{tabular}

Table 21 Histological classification

\begin{tabular}{lr}
\hline Histological classification & \multicolumn{1}{c}{ Cases $(\%)$} \\
\hline Squamous cell carcinoma & $3300(86.7 \%)$ \\
$\quad$ Squamous cell carcinoma & $685(18.0 \%)$ \\
Well differentiated & $653(17.2 \%)$ \\
Moderately differentiated & $1521(40.0 \%)$ \\
Poorly differentiated & $441(11.6 \%)$ \\
Adenocarcinoma & $222(5.8 \%)$ \\
Adenosquamous cell carcinoma & $35(0.9 \%)$ \\
Adenoid cystic carcinoma & $1(0.0 \%)$ \\
Basaloid carcinoma & $56(1.5 \%)$ \\
Neuroendocrine cell carcinoma & $17(0.4 \%)$ \\
Undifferentiated carcinoma & $10(0.3 \%)$ \\
Other carcinoma & $9(0.2 \%)$ \\
Carcinosarcoma & $21(0.6 \%)$ \\
Malignant melanoma & $11(0.3 \%)$ \\
GIST & $1(0.0 \%)$ \\
Other & $46(1.2 \%)$ \\
Unknown & $78(2.0 \%)$ \\
Total & $3807(100 \%)$ \\
\hline
\end{tabular}

Table 22 Depth of tumor invasion, pT (JES 10th)

\begin{tabular}{lc}
\hline pT category & Cases $(\%)$ \\
\hline pTX & $24(0.6 \%)$ \\
pT0 & $94(2.4 \%)$ \\
pTis & $29(0.8 \%)$ \\
pT1a & $422(11.0 \%)$ \\
pT1b & $1065(27.7 \%)$ \\
pT2 & $454(11.8 \%)$ \\
pT3 & $1518(39.5 \%)$ \\
pT4 & $127(3.3 \%)$ \\
pT4a & $27(0.7 \%)$ \\
pT4b & $30(0.8 \%)$ \\
Unknown & $54(1.4 \%)$ \\
Total & $3844(100 \%)$ \\
\hline
\end{tabular}

Table 23 Pathological grading of lymph node metastasis, pN (JES 10th)

\begin{tabular}{lc}
\hline Lymph node metastasis & Cases $(\%)$ \\
\hline pN0 & $2270(59.1 \%)$ \\
pN1 & $492(12.8 \%)$ \\
pN2 & $584(15.2 \%)$ \\
pN3 & $225(5.9 \%)$ \\
pN4 & $185(4.8 \%)$ \\
Unknown & $88(2.3 \%)$ \\
Total & $3844(100 \%)$ \\
\hline
\end{tabular}

Table 24 Numbers of the metastatic nodes

\begin{tabular}{lc}
\hline Numbers of lymph node metastasis & Cases $(\%)$ \\
\hline 0 & $1779(46.3 \%)$ \\
$1-2$ & $985(25.6 \%)$ \\
$3-6$ & $640(16.6 \%)$ \\
$7-$ & $376(9.8 \%)$ \\
Unknown & $64(1.7 \%)$ \\
Total & $3844(100 \%)$ \\
\hline
\end{tabular}

Table 25 Pathological findings of distant organ metastasis, pM (JES 10th)

\begin{tabular}{lc}
\hline Distant metastasis & Cases $(\%)$ \\
\hline pMX & $53(1.4 \%)$ \\
pM0 & $3733(97.1 \%)$ \\
pM1 & $58(1.5 \%)$ \\
Total & $3844(100 \%)$ \\
\hline
\end{tabular}


Table 26 Residual tumor, R

\begin{tabular}{lc}
\hline Residual tumor & Cases $(\%)$ \\
\hline RX & $156(4.1 \%)$ \\
R0 & $3345(87.0 \%)$ \\
R1 & $187(4.9 \%)$ \\
R2 & $156(4.1 \%)$ \\
Total & $3844(100 \%)$ \\
\hline
\end{tabular}

Table 27 Causes of death

\begin{tabular}{lc}
\hline Cause of death & Cases $(\%)$ \\
\hline Death due to recurrence & $1139(72.8 \%)$ \\
Death due to other cancer & $65(4.2 \%)$ \\
Death due to other disease (rec +) & $44(2.8 \%)$ \\
Death due to other disease (rec-) & $179(11.4 \%)$ \\
Death due to other disease (rec?) & $7(0.4 \%)$ \\
Operative death* & $39(2.5 \%)$ \\
Postoperative hospital death** & $40(2.6 \%)$ \\
Unknown & $52(3.3 \%)$ \\
Total of death cases & $1565(100 \%)$
\end{tabular}

rec recurrence

* Operative death means death within 30 days after operation in or out of hospital

** Hospital death is defined as death during the same hospitalization, regardless of department at time of death

Operative mortality after esophagectomy: $1.01 \%$

Hospital mortality after esophagectomy: $4.76 \%$

Follow-up period (months)

Median (min - max) $41.13(0.00$ - 71.03)
Fig. 6 Survival of patients who underwent esophagectomy

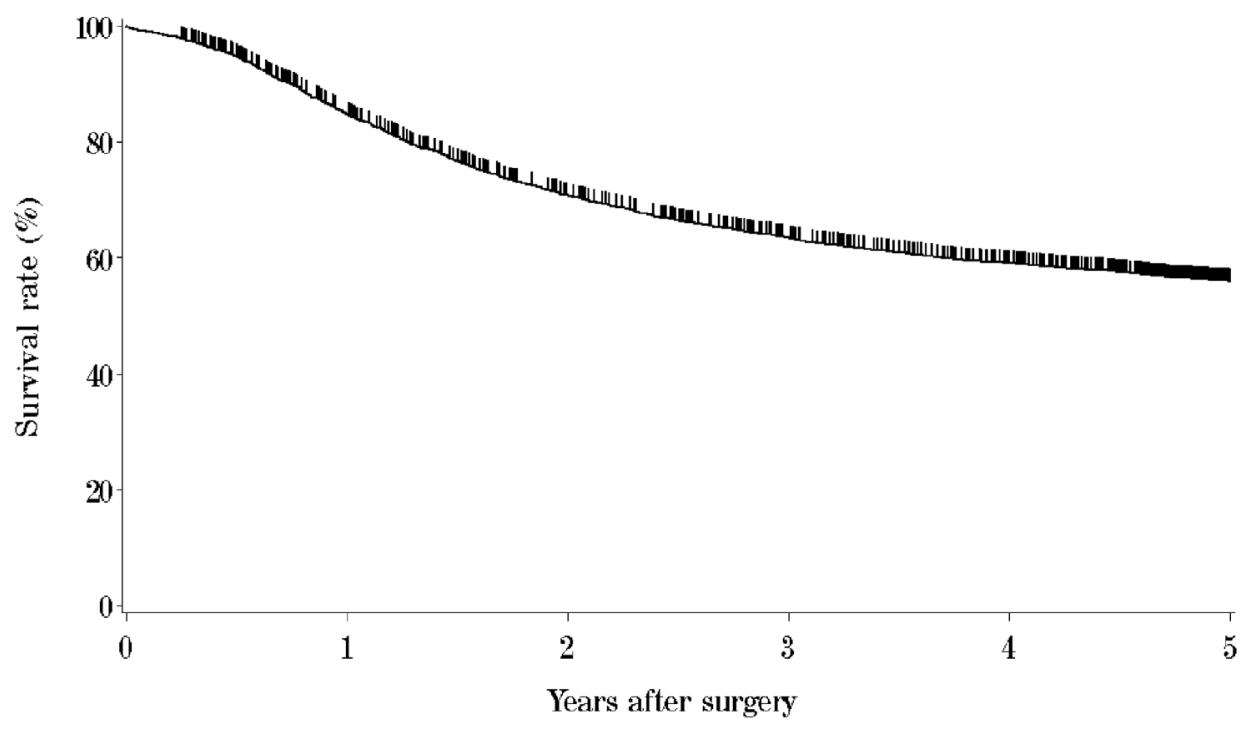

Esophagectomy $(n=3612)$

\begin{tabular}{lccccc}
\hline & \multicolumn{5}{c}{ Years after surgery } \\
\cline { 2 - 6 } & 1 & 2 & 3 & 4 & 5 \\
\hline Esophagectomy & $\mathbf{8 4 . 9 \%}$ & $\mathbf{7 0 . 8 \%}$ & $\mathbf{6 3 . 5 \%}$ & $59.1 \%$ & $55.9 \%$ \\
\hline
\end{tabular}


Fig. 7 Survival of patients who underwent esophagectomy according to clinical stage (JES TNM 10th)

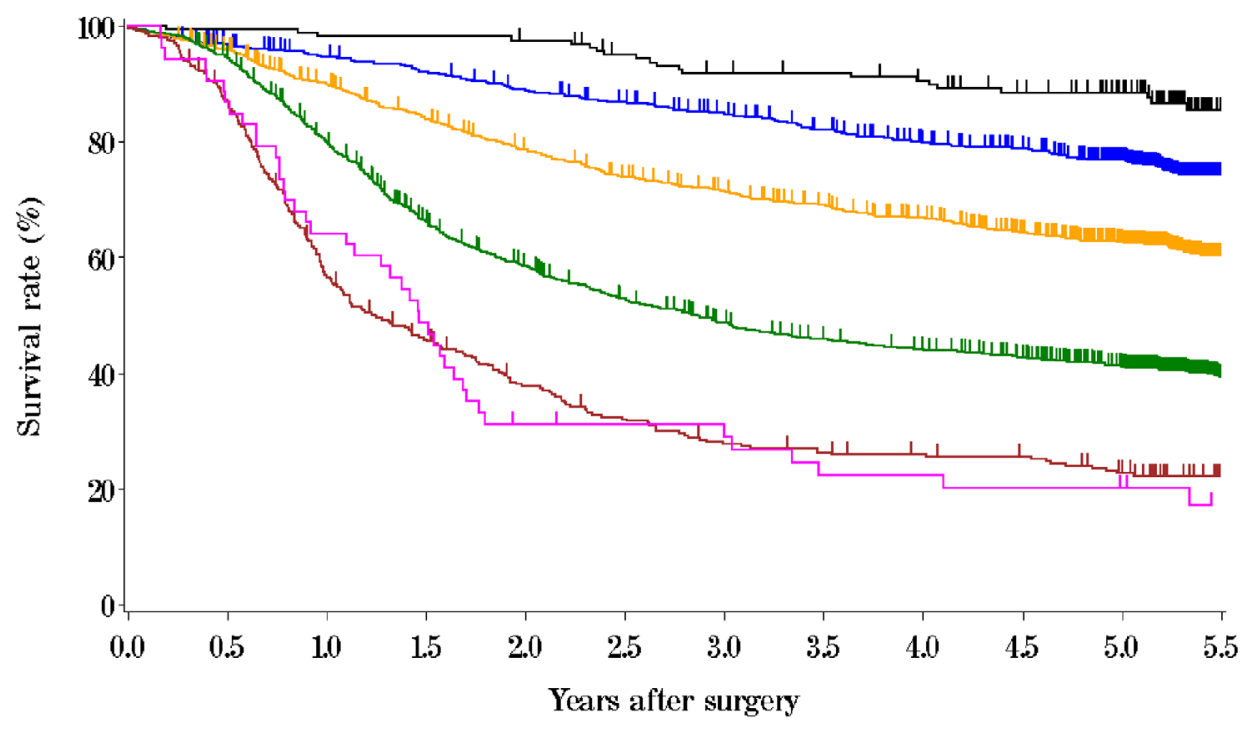

$\begin{array}{lll}\text { cStage } 0(n=171) & \text { cStage } I(n=762) & \text { cStage II }(n=1021) \\ \text { cStage } \text { III }(n=1170) & \text { cStage IVA }(n=308) & \text { cStage IVB }(n=55)\end{array}$

\begin{tabular}{lccccc}
\hline & \multicolumn{5}{c}{ Years after surgery } \\
\cline { 2 - 6 } & 1 & 2 & 3 & 4 & $58.6 \%$ \\
\hline cStage 0 & $98.2 \%$ & $97.6 \%$ & $91.9 \%$ & $90.6 \%$ & $76.8 \%$ \\
cStage I & $94.8 \%$ & $89.0 \%$ & $85.0 \%$ & $79.9 \%$ & $62.7 \%$ \\
cStage III & $90.2 \%$ & $78.7 \%$ & $71.5 \%$ & $66.9 \%$ & $41.2 \%$ \\
cStage IVA & $80.0 \%$ & $58.4 \%$ & $48.6 \%$ & $44.0 \%$ & $22.6 \%$ \\
cStage IVB & $56.9 \%$ & $37.7 \%$ & $27.7 \%$ & $25.8 \%$ & $20.0 \%$ \\
\hline
\end{tabular}


Fig. 8 Survival of patients who underwent esophagectomy according to clinical stage (UICC TNM 6th)

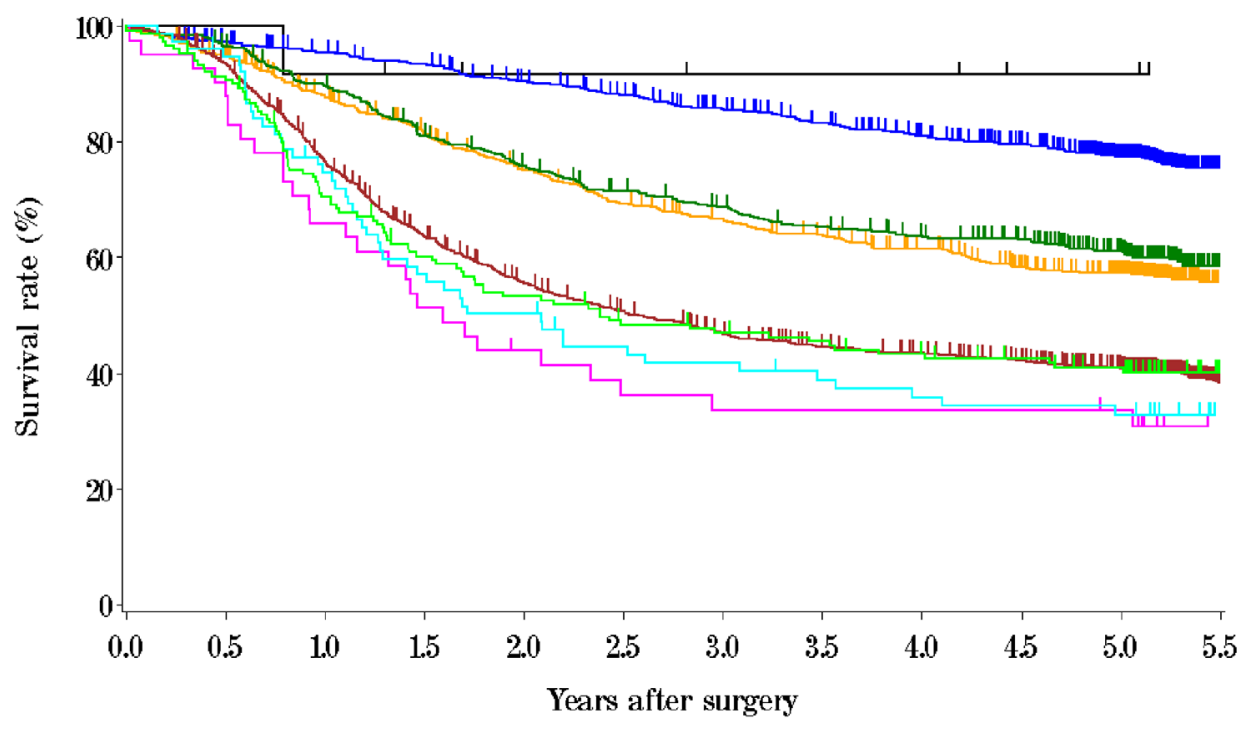

$\begin{array}{lll}\text { cStage } 0(n=13) & \text { cStage I }(n=911) & \text { cStage IIA }(n=676) \\ \text { cStage IIB }(n=515) & \text { cStage III }(n=1166) \\ \text { cStage IVA }(n=76) & \text { cStage IVB }(n=151)\end{array}$

\begin{tabular}{lccccc}
\hline & \multicolumn{5}{c}{ Years after surgery } \\
\cline { 2 - 6 } & 1 & 2 & 3 & 4 & 5 \\
\hline cStage 0 & $91.7 \%$ & $91.7 \%$ & $91.7 \%$ & $91.7 \%$ & $91.7 \%$ \\
cStage I & $95.4 \%$ & $90.5 \%$ & $85.8 \%$ & $81.0 \%$ & $77.5 \%$ \\
cStage IIA & $88.1 \%$ & $75.3 \%$ & $66.7 \%$ & $61.6 \%$ & $57.4 \%$ \\
cStage IIB & $89.7 \%$ & $75.9 \%$ & $68.7 \%$ & $63.7 \%$ & $61.1 \%$ \\
cStage III & $76.8 \%$ & $55.6 \%$ & $46.7 \%$ & $43.5 \%$ & $40.7 \%$ \\
cStage IV & $65.9 \%$ & $43.9 \%$ & $33.6 \%$ & $33.6 \%$ & $33.6 \%$ \\
cStage IVA & $74.7 \%$ & $50.2 \%$ & $41.7 \%$ & $35.8 \%$ & $32.3 \%$ \\
cStage IVB & $70.4 \%$ & $53.3 \%$ & $46.9 \%$ & $43.2 \%$ & $40.9 \%$ \\
\hline
\end{tabular}


Fig. 9 Survival of patients who underwent esophagectomy according to the depth of tumor invasion (JES 10th: pT)

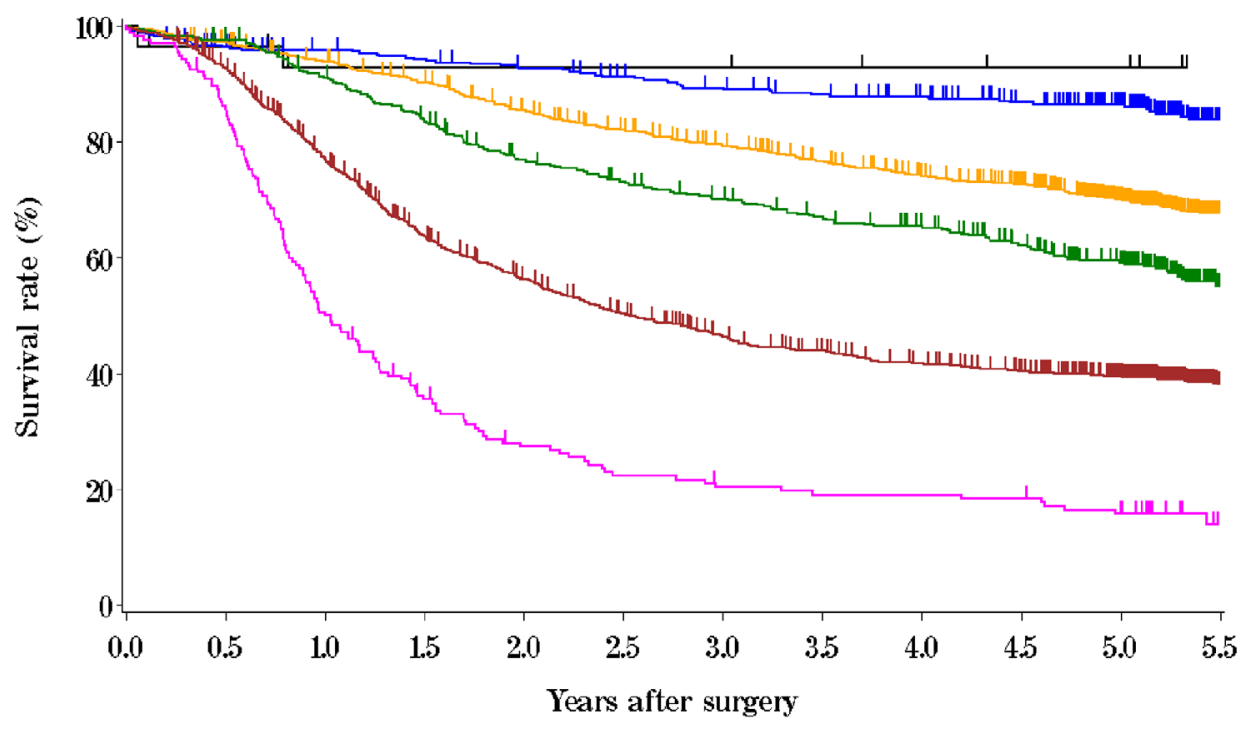

$$
\begin{array}{lll}
\text { pTis }(n=29) & \text { pT1a }(n=394) & \text { pT1b }(n=1015) \\
\text { pT2 }(n=424) & \text { pT3 }(n=1416) & \text { pT4 }(n=175)
\end{array}
$$

\begin{tabular}{lccccc}
\hline & \multicolumn{5}{c}{ Years after surgery } \\
\cline { 2 - 6 } & 1 & 2 & 3 & 4 & 5 \\
\hline pTis & $92.8 \%$ & $92.8 \%$ & $92.8 \%$ & $92.8 \%$ & $92.8 \%$ \\
pT1a & $95.9 \%$ & $92.7 \%$ & $89.4 \%$ & $88.0 \%$ & $86.5 \%$ \\
pT1b & $93.9 \%$ & $85.5 \%$ & $79.6 \%$ & $74.2 \%$ & $70.0 \%$ \\
pT2 & $91.1 \%$ & $76.9 \%$ & $70.1 \%$ & $65.4 \%$ & $59.1 \%$ \\
pT3 & $77.2 \%$ & $56.3 \%$ & $46.4 \%$ & $41.6 \%$ & $39.4 \%$ \\
pT4 & $50.6 \%$ & $27.3 \%$ & $20.3 \%$ & $19.0 \%$ & $15.6 \%$ \\
\hline
\end{tabular}


Fig. 10 Survival of patients who underwent esophagectomy according to the depth of tumor invasion (UICC TNM 6th: pT)

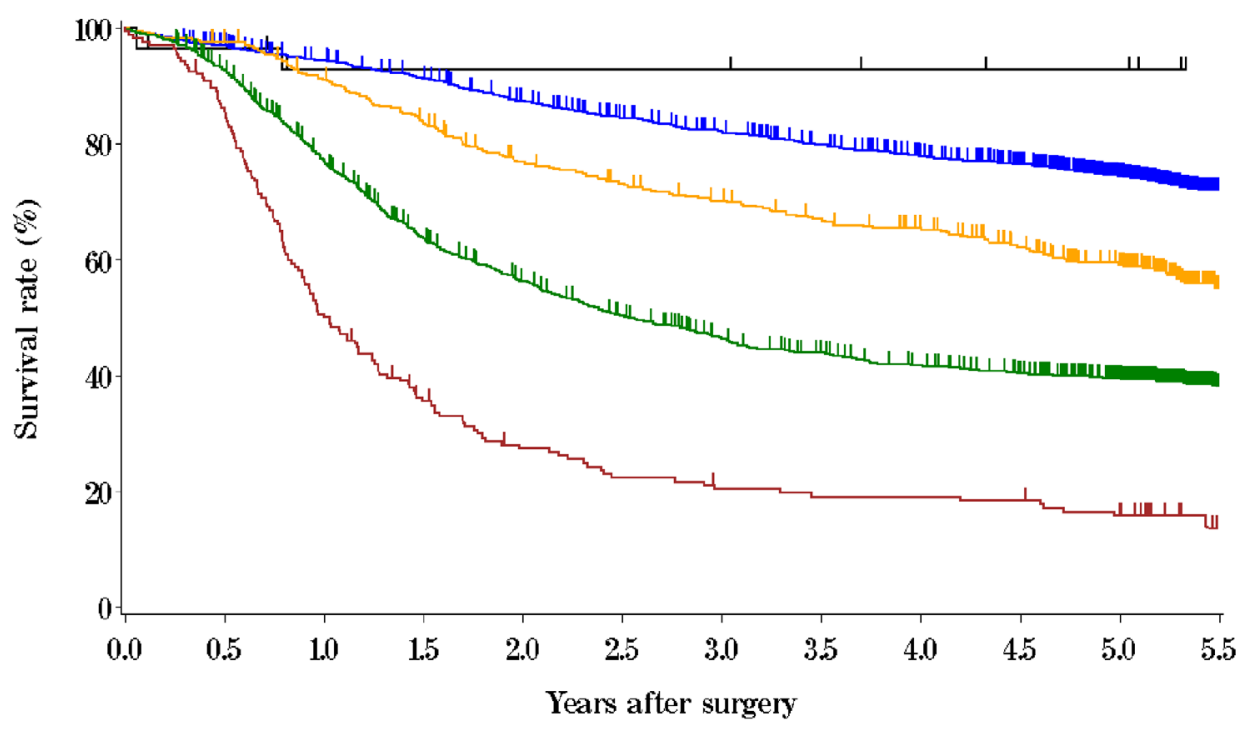

$\begin{array}{ll}\text { pTis }(n=29) & \operatorname{pT} 1(n=1409) \\ \text { pT3 }(n=1416) & \text { pT4 }(n=175)\end{array}$

\begin{tabular}{|c|c|c|c|c|c|}
\hline & \multicolumn{5}{|c|}{ Years after surgery } \\
\hline & 1 & 2 & 3 & 4 & 5 \\
\hline pTis & $92.8 \%$ & $92.8 \%$ & $92.8 \%$ & $92.8 \%$ & $92.8 \%$ \\
\hline pT1 & $94.4 \%$ & $87.5 \%$ & $82.3 \%$ & $78.0 \%$ & $74.6 \%$ \\
\hline pT2 & $91.1 \%$ & $76.9 \%$ & $70.1 \%$ & $65.4 \%$ & $59.1 \%$ \\
\hline pT3 & $77.2 \%$ & $56.3 \%$ & $46.4 \%$ & $41.6 \%$ & $39.4 \%$ \\
\hline pT4 & $50.6 \%$ & $27.3 \%$ & $20.3 \%$ & $19.0 \%$ & $15.6 \%$ \\
\hline
\end{tabular}


Fig. 11 Survival of patients who underwent esophagectomy according to lymph node metastasis (JES 10th: pN)

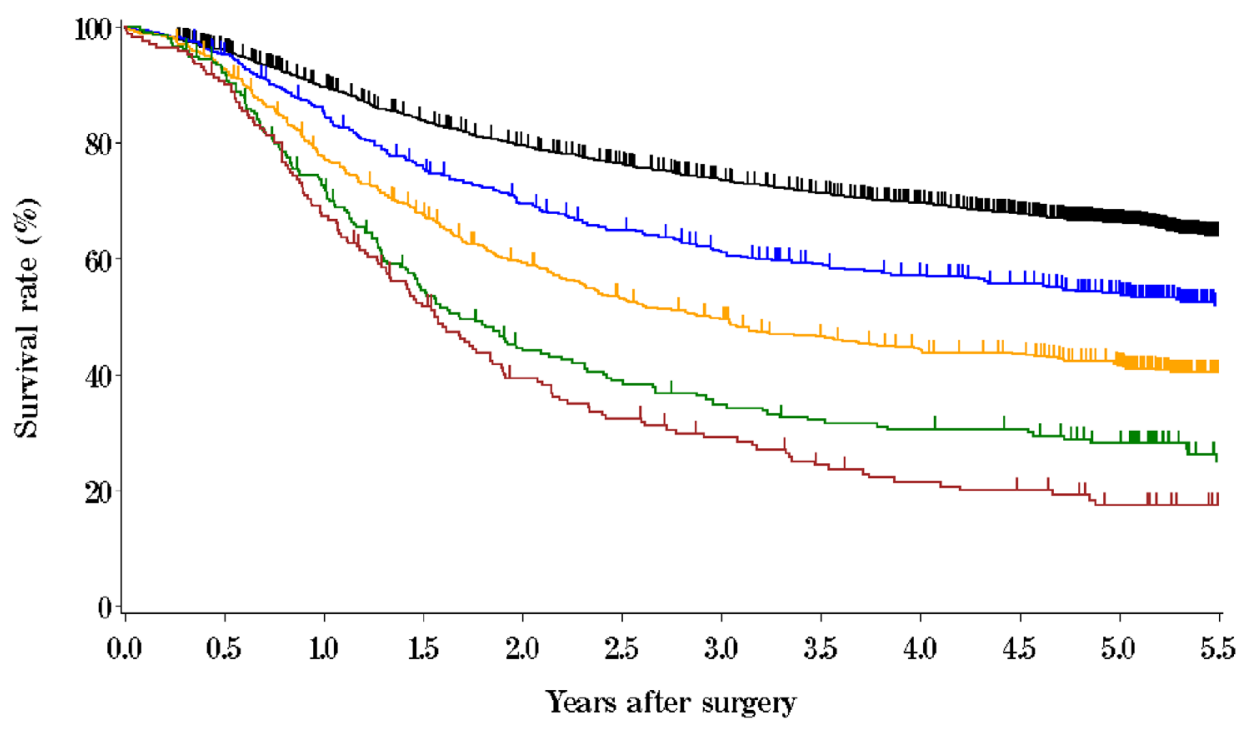

$\begin{array}{ll}\text { pN0 }(n=2135) & \text { pN1 }(n=327) \\ \text { pN3 }(n=145) & \text { pN4 }(n=110)\end{array}$

\begin{tabular}{lccccc}
\hline & \multicolumn{5}{c}{ Years after surgery } \\
\cline { 2 - 6 } & 1 & 2 & 3 & 4 & 5 \\
\hline pN0 & $89.7 \%$ & $79.6 \%$ & $73.7 \%$ & $69.7 \%$ & $66.3 \%$ \\
pN1 & $85.5 \%$ & $69.5 \%$ & $61.0 \%$ & $57.2 \%$ & $54.0 \%$ \\
pN3 & $77.7 \%$ & $59.3 \%$ & $49.4 \%$ & $44.2 \%$ & $41.5 \%$ \\
pN4 & $72.7 \%$ & $44.5 \%$ & $34.7 \%$ & $30.4 \%$ & $28.1 \%$ \\
\hline
\end{tabular}


Fig. 12 Survival of patients who underwent esophagectomy according to lymph node metastasis (UICC TNM 6th: pN)

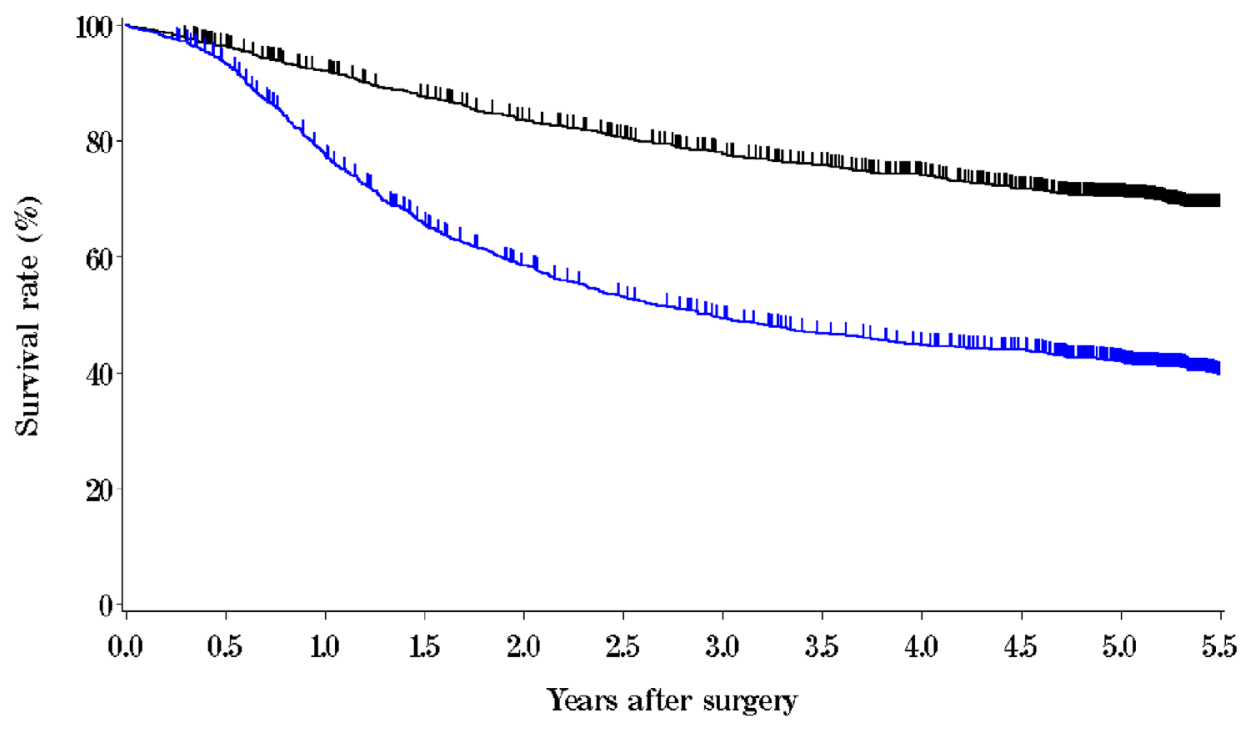

$\longrightarrow$ pN0 $(n=494) \quad$ pN1 $(n=1491)$

\begin{tabular}{lcccccc}
\hline & \multicolumn{5}{c}{ Years aftersurgery } \\
\cline { 2 - 6 } & 1 & 2 & 3 & 4 & 5 \\
\hline pN0 & $92.0 \%$ & $83.7 \%$ & $77.9 \%$ & $74.1 \%$ & $70.5 \%$ \\
pN1 & $77.9 \%$ & $58.5 \%$ & $49.4 \%$ & $44.7 \%$ & $41.9 \%$ \\
\hline
\end{tabular}


Fig. 13 Survival of patients who underwent esophagectomy according to number of metastatic nodes

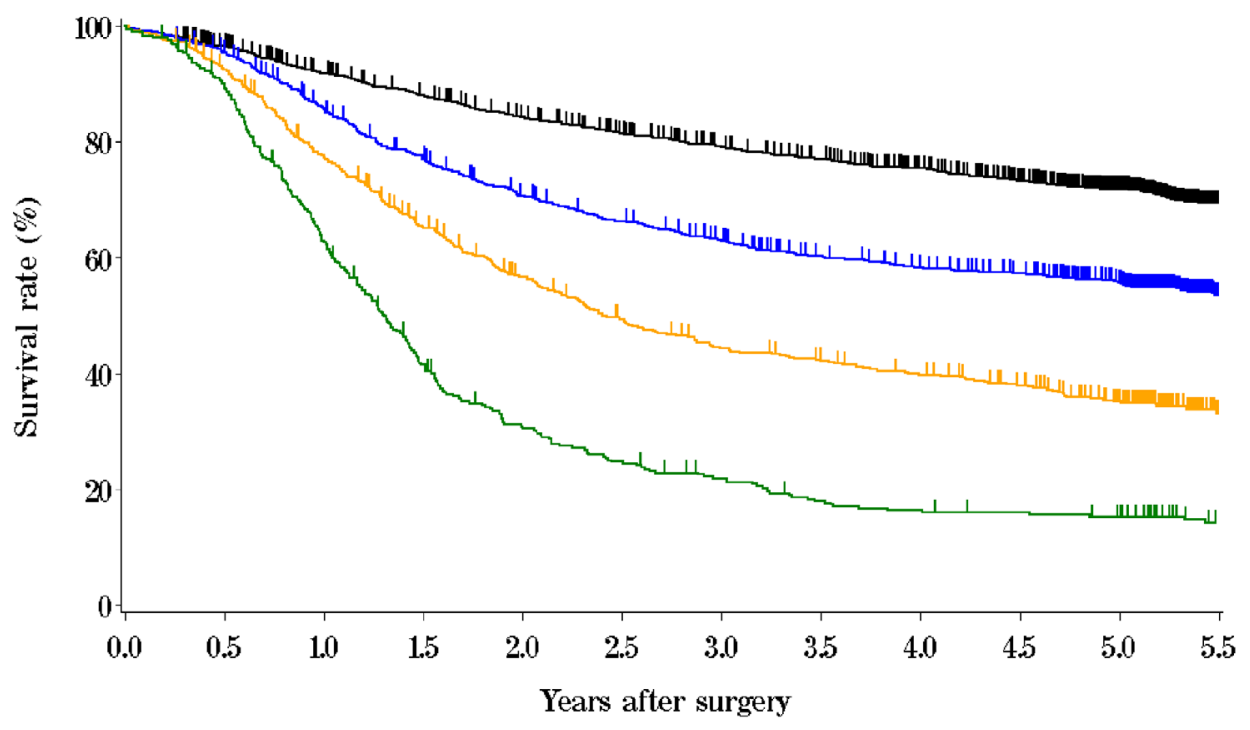

$\mathbf{0}(\mathrm{n}=1687) \quad$ 1-2 $(n=937) \quad 3-7(n=591) \quad$ - $\quad$ - $(n=348)$

\begin{tabular}{lccccc}
\hline & \multicolumn{5}{c}{ Years after surgery } \\
\cline { 2 - 6 } & 1 & 2 & 3 & 4 & 5 \\
\hline 0 & $91.9 \%$ & $84.3 \%$ & $79.3 \%$ & $75.4 \%$ & $71.9 \%$ \\
$1-2$ & $85.8 \%$ & $70.7 \%$ & $62.9 \%$ & $58.4 \%$ & $55.7 \%$ \\
$7-6$ & $77.6 \%$ & $56.6 \%$ & $44.4 \%$ & $39.8 \%$ & $35.2 \%$ \\
\hline
\end{tabular}


Fig. 14 Survival of patients who underwent esophagectomy according to pathological stage (JES 10th)
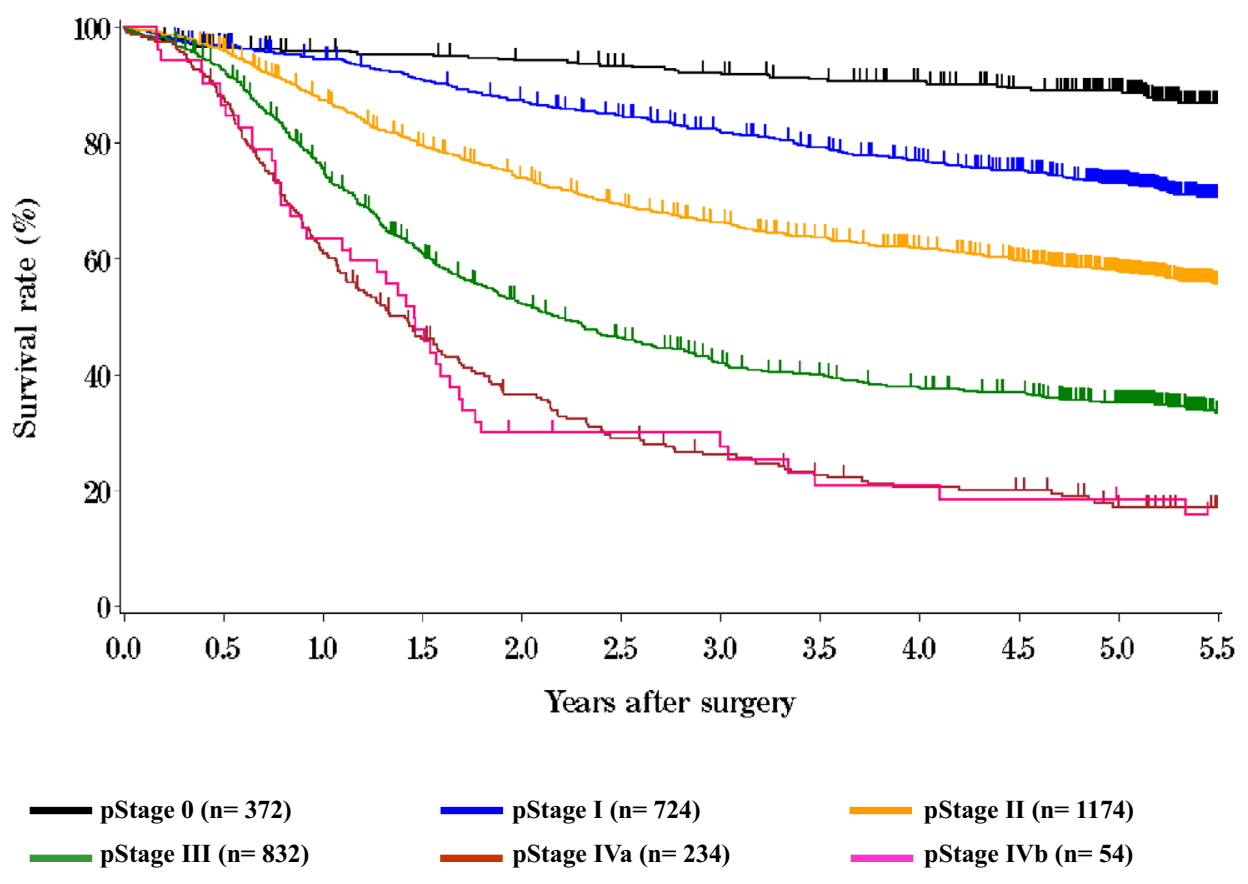

\begin{tabular}{lccccc}
\hline & \multicolumn{3}{c}{ Years after surgery } \\
\cline { 2 - 6 } & 1 & 2 & 3 & 4 & $\mathbf{8 9 . 1} \%$ \\
\hline pStage 0 & $95.9 \%$ & $94.2 \%$ & $92.2 \%$ & $90.7 \%$ & $73.1 \%$ \\
pStage I & $94.5 \%$ & $87.5 \%$ & $82.2 \%$ & $76.9 \%$ & $57.8 \%$ \\
pStage II & $87.4 \%$ & $74.0 \%$ & $66.3 \%$ & $61.8 \%$ & $35.2 \%$ \\
pStage III & $75.7 \%$ & $52.2 \%$ & $41.8 \%$ & $37.7 \%$ & $17.0 \%$ \\
pStage IVa & $61.4 \%$ & $36.4 \%$ & $26.1 \%$ & $20.5 \%$ & $18.3 \%$ \\
pStage IVb & $63.5 \%$ & $29.8 \%$ & $27.5 \%$ & $20.6 \%$ &
\end{tabular}


Fig. 15 Survival of patients who underwent esophagectomy according to pathological stage (UICC TNM 6th)
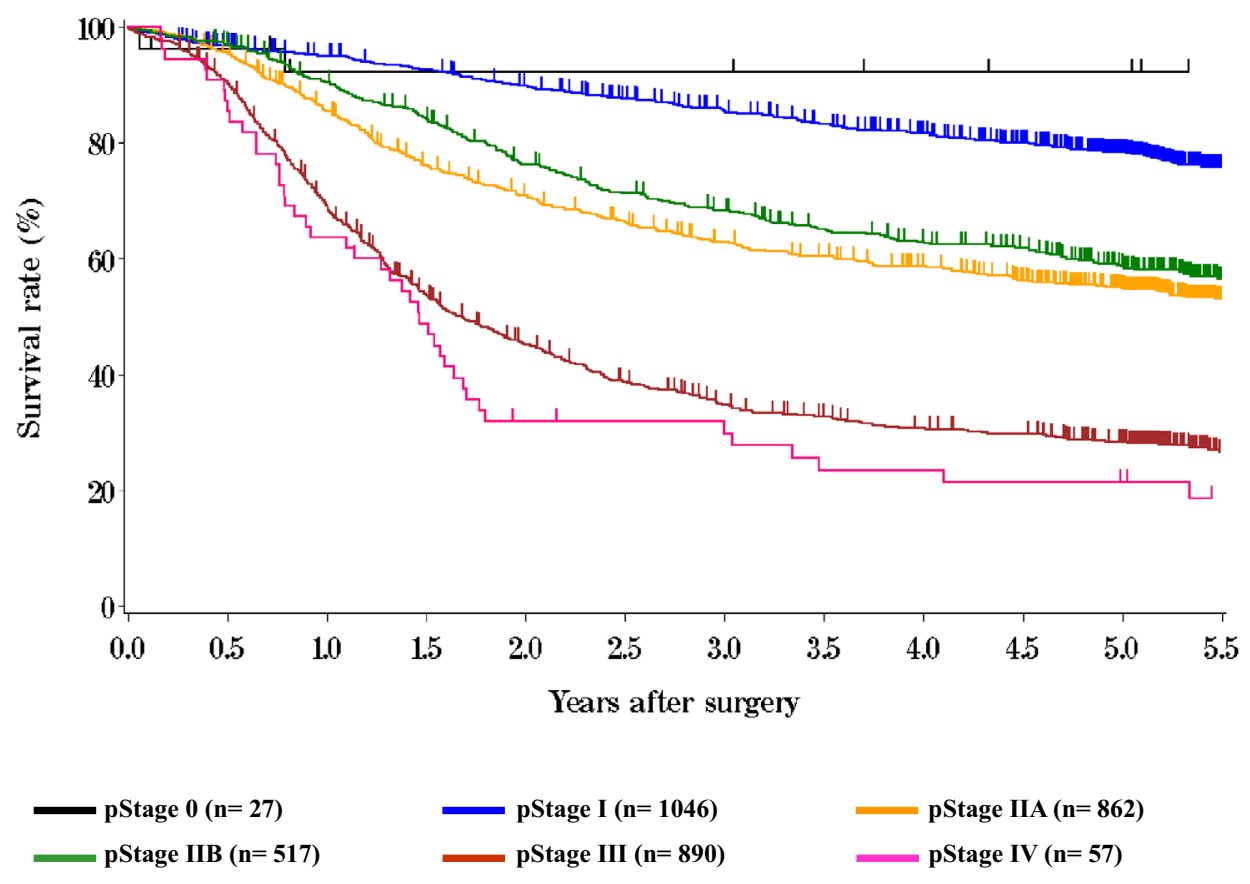

\begin{tabular}{lccccc}
\hline & \multicolumn{5}{c}{ Years after surgery } \\
\cline { 2 - 6 } & 1 & 2 & 3 & 4 & 5 \\
\hline pStage 0 & $92.2 \%$ & $92.2 \%$ & $92.2 \%$ & $92.2 \%$ & $92.2 \%$ \\
pStage I & $95.0 \%$ & $90.0 \%$ & $85.8 \%$ & $81.6 \%$ & $78.4 \%$ \\
pStage IIA & $85.6 \%$ & $70.8 \%$ & $62.9 \%$ & $58.7 \%$ & $54.9 \%$ \\
pStage IIB & $90.6 \%$ & $76.3 \%$ & $68.3 \%$ & $62.9 \%$ & $58.6 \%$ \\
pStage III & $69.4 \%$ & $45.2 \%$ & $34.7 \%$ & $30.6 \%$ & $28.2 \%$ \\
pStage IV & $63.6 \%$ & $31.9 \%$ & $29.8 \%$ & $23.4 \%$ & $21.3 \%$ \\
\hline
\end{tabular}


Fig. 16 Survival of patients who underwent esophagectomy according to residual tumor $(\mathrm{R})$

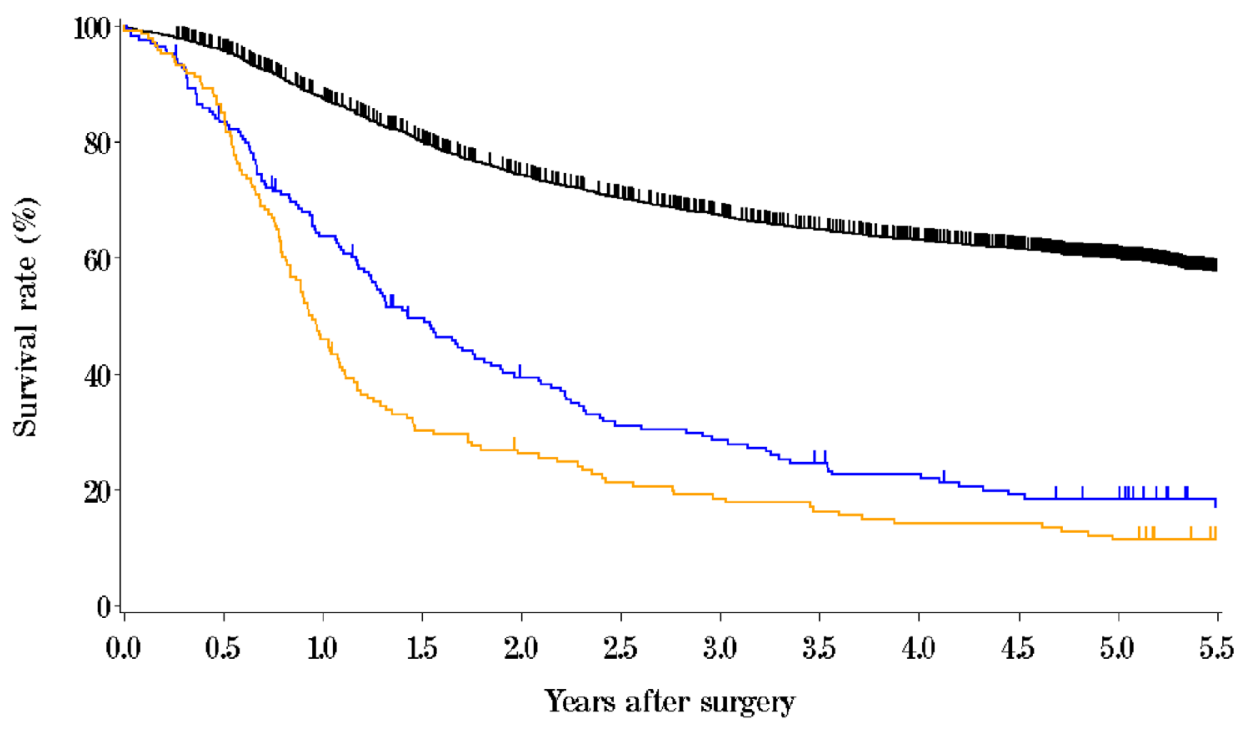

$\operatorname{R0}(\mathrm{n}=3152) \quad \mathrm{R} 1(\mathrm{n}=170) \quad \mathrm{R} 2(\mathrm{n}=148)$

\begin{tabular}{lccccc}
\hline & \multicolumn{5}{c}{ Years after surgery } \\
\cline { 2 - 6 } & 1 & 2 & 3 & 4 & 5 \\
\hline R0 & $87.7 \%$ & $74.3 \%$ & $67.4 \%$ & $63.1 \%$ & $60.0 \%$ \\
R1 & $63.6 \%$ & $39.4 \%$ & $28.4 \%$ & $22.5 \%$ & $18.3 \%$ \\
R2 & $46.0 \%$ & $26.1 \%$ & $18.3 \%$ & $14.1 \%$ & $11.3 \%$ \\
\hline
\end{tabular}

Acknowledgments This study was supported by Health and Labour Sciences Research Grants for Promotion of Cancer Control Programs (H26-Cancer Policy-General-014) from the Ministry of Health, Labour and Welfare of Japan.

\section{Compliance with ethical standards}

Ethical Statement All procedures were in accordance with the ethical standards of the responsible committee on human experimentation (institutional and national) and with the Helsinki Declaration of 1964 and later versions.
Conflict of interest All authors have nothing to disclose with regard to commercial support.

Open Access This article is distributed under the terms of the Creative Commons Attribution 4.0 International License (http://creativecommons.org/licenses/by/4.0/), which permits unrestricted use, distribution, and reproduction in any medium, provided you give appropriate credit to the original author(s) and the source, provide a link to the Creative Commons license, and indicate if changes were made. 